\title{
An Ireland-Claisen Approach to Lignans: Synthesis of 5-epi-Eupomatilone-6
}

John M. Hutchison, Sang-phyo Hong and Matthias C. McIntosh

\section{Supporting Information}

\section{Contents}

Experimental Data for compounds 14, 12, $11(\operatorname{method} 1), 15,16,11(\operatorname{method} 2)$,

36, 37, 38, 39, and 9:

Epxoyketal 14

Bromoepoxyenone 12

Ester 11 (method 1)

Alcohol 15

Alcohol 16

Ester 11 (method 2)

Methyl Ester 36

S6

Diol 37

S6

Epoxyalcohol 38

S7

Epoxyketone 39

S7

o-Quinonemonoketal 9

S8 
${ }^{1} \mathrm{H}$ NMR and ${ }^{13} \mathrm{C}$ NMR spectra of compounds 14, 12, 11, 15, 16, 10, 17, 23, 29, $32,34,35,36,37,38,39$ and 9 :

Epoxyketal 14

S9-S10

Bromoepoxyenone 12

S11-S12

Ester 11 (method 1)

S13-S14

Alcohol 15

S15-S16

Alcohol 16

S17-S18

Acid 10

S19-S20

Lactone 5-epi-17

S21-S22

o-Quinonemonoketal 23

S23-S24

Arylketal 29

S25-S26

Arylepoxyenone 32

S27-S28

Hydroxyenone 33 ( ${ }^{1} \mathrm{H}$ NMR only)

S29

Phenol 34 ( ${ }^{1} \mathrm{H}$ NMR only)

S30

5-epi-eupomatilone 35

S31-S32

Methyl Ester 36

S33-S34

Diol 37

S35-S36

Epoxyalcohol 38

S37-S38

Epoxyketone 39

S39-S40

o-Quinonemonoketal 9

S41-S42 
General Experimental Procedures: THF, ether and $\mathrm{CH}_{2} \mathrm{Cl}_{2}$ were purified by passage through activated alumina. ${ }^{1} \mathrm{H}$ and ${ }^{13} \mathrm{C}$ NMR spectra were obtained using 270 or 300 $\mathrm{MHz}$ spectrometers. All spectra were obtained using $\mathrm{CDCl}_{3}$ unless otherwise noted. All reactions were performed under an atmosphere of $\mathrm{N}_{2}$.

Epoxy ketal 14. $\mathrm{K}_{2} \mathrm{CO}_{3}(2.7 \mathrm{~g}, 19.5 \mathrm{mmol})$ was added to a solution of monoketal 13 (1.0 g, $6.5 \mathrm{mmol})$ in THF $(30 \mathrm{~mL})$ and water $(30 \mathrm{~mL}) . \mathrm{H}_{2} \mathrm{O}_{2}(1.15 \mathrm{ml}, 30 \%$ in water) was added and the resulting mixture allowed to warm to rt and stir for $16 \mathrm{~h}$. After an extractive workup with ether, the residue was subject to microdistillation to give monoketal $3(0.40 \mathrm{~g})$ and epoxy ketal 14 as a yellow oil (0.28 g, 25\%): IR (film) 2944(m), $1700 \mathrm{~cm}^{-1} ;{ }^{1} \mathrm{H}$ NMR (270 MHz, $\left.\mathrm{CDCl}_{3}\right) 3.32$ (s, 3H), 3.42 (dd, $J=3.96,1.98$ Hz, 1H), 3.48 (s, 3H), 3.74 (dd, $J=3.96,2.97 \mathrm{~Hz}, 1 \mathrm{H}), 5.93$ (dd, $J=10.89,1.98 \mathrm{~Hz}$, 1H), $6.49(\mathrm{dd}, J=10.89,2.97 \mathrm{~Hz}, 1 \mathrm{H}) ;{ }^{13} \mathrm{C} \mathrm{NMR}\left(67 \mathrm{MHz}, \mathrm{CDCl}_{3}\right) 49.7,49.8,52.1$, 53.8, 94.8, 126.7, 142.5, 192.8; MS (m/z) 155, 139, 123, 111, 95, 88, 68, 55, 39, 29.

Bromoepoxy enone 12. Bromine $(0.085 \mathrm{~mL}, 1.65 \mathrm{mmol})$ was added to a solution of epoxy ketal $4(0.28 \mathrm{~g}, 1.65 \mathrm{mmol})$ in ether $(50 \mathrm{~mL})$ and hexane $(50 \mathrm{~mL})$. After $3 \mathrm{~h}$ the mixture was treated with $\mathrm{Et}_{3} \mathrm{~N}(0.35 \mathrm{~mL}, 2.48 \mathrm{mmol})$. After $1 \mathrm{~h}$ the mixture was filtered and the filtrate concentrated in vacuo. The residue was purified by flash chromatography on silica gel with 20/80 ethyl acetate/hexane to give bromoepoxy enone 5 as white solid (0.35 g, 84\%): IR (KBr) 2942(m), $1700 \mathrm{~cm}^{-1} ;{ }^{1} \mathrm{H} \mathrm{NMR}\left(270 \mathrm{MHz}, \mathrm{CDCl}_{3}\right) 3.38$ (s, 3H), $3.51(\mathrm{~s}, 3 \mathrm{H}), 3.64(\mathrm{~d}, J=3.96 \mathrm{~Hz}, 1 \mathrm{H}), 3.79(\mathrm{dd}, J=3.96,2.77 \mathrm{~Hz}, 1 \mathrm{H}), 6.98(\mathrm{~d}, J=$ 
$2.77 \mathrm{~Hz}, 1 \mathrm{H}) ;{ }^{13} \mathrm{C}$ NMR $\left(67 \mathrm{MHz}, \mathrm{CDCl}_{3}\right) 50.0,50.2,51.6,53.8,96.3,122.5,143.0$, 186.3.

Ester 11. (Method 1): t-BuLi (14.0 $\mathrm{mL}, 1.7 \mathrm{M}$ in pentane, $23.8 \mathrm{mmol}$ ) was added dropwise to a solution of trans-1-propenylbromide $(1.03 \mathrm{ml}, 24.0 \mathrm{mmol})$ in THF (100 $\mathrm{mL})$ at $-78{ }^{\circ} \mathrm{C}$, followed after $15 \mathrm{~min}$ by bromoepoxy enone $12(2.0 \mathrm{~g}, 8.0 \mathrm{mmol})$ in THF $(30 \mathrm{~mL})$. The reaction mixture was allowed to warm to $0{ }^{\circ} \mathrm{C}$ over $20 \mathrm{~min}$. Propionic anhydride (3.1 ml, $24.2 \mathrm{mmol}$ ) was added and the mixture allowed to warm to rt. After extractive workup with ether, the residue was purified by flash chromatography on Florisil with 20/80 ethyl acetate/hexane to give ester 11 as yellow oil (2.7 g, 97\%): IR (film) $2942(\mathrm{~m}) 1742 \mathrm{~cm}^{-1} ;{ }^{1} \mathrm{H}$ NMR $\left(270 \mathrm{MHz}, \mathrm{CDCl}_{3}\right) 1.51(\mathrm{t}, J=7.52 \mathrm{~Hz}, 3 \mathrm{H}), 1.75$ (d, $J=4.95 \mathrm{~Hz}, 3 \mathrm{H}), 2.42$ (q, $J=7.52 \mathrm{~Hz}, 2 \mathrm{H}), 3.31(\mathrm{~s}, 3 \mathrm{H}), 3.44(\mathrm{~s}, 3 \mathrm{H}), 3.51$ (dd, $J=$ $4.35,2.38 \mathrm{~Hz}, 1 \mathrm{H}), 4.20(\mathrm{~d}, J=4.35 \mathrm{~Hz}, 1 \mathrm{H}), 5.68-5.78(\mathrm{~m}, 2 \mathrm{H}), 6.09$ (d, $J=2.57 \mathrm{~Hz}$, $1 \mathrm{H}) ;{ }^{13} \mathrm{C} \mathrm{NMR}\left(67 \mathrm{MHz}, \mathrm{CDCl}_{3}\right)$ 9.1, 18.2, 28.4, 49.6, 49.7, 53.0, 53.9, 80.2, 96.7, 127.1, $127.8,129.8,172.6$.

Alcohol 15. PropynylMgBr (16.1 ml, $0.5 \mathrm{M}$ in THF, $8.05 \mathrm{mmol})$ was added dropwise to a solution of bromoepoxy enone $12(1.00 \mathrm{~g}, 4.03 \mathrm{mmol})$ in ether $(75 \mathrm{ml})$ at $0{ }^{\circ} \mathrm{C}$. After $2.5 \mathrm{~h}$, reaction mixture was allowed to warm to $\mathrm{rt}$ and the resulting mixture poured into saturated $\mathrm{NaHCO}_{3}$ solution. After extractive workup with ether, the residue was purified by flash chromatography on silica gel with 10/90 ethyl acetate/hexane, followed by 20/80 ethyl acetate/hexane to give alcohol $\mathbf{1 5}$ as a white solid $(1.00 \mathrm{~g}, 86 \%)$ : $\mathrm{mp} 112-113{ }^{\circ} \mathrm{C}$; IR (film) 3411 (br), $2240 \mathrm{~cm}^{-1}$; ${ }^{1} \mathrm{H}$ NMR (270 MHz, $\left.\mathrm{CDCl}_{3}\right) 1.87$ (s, 3H), 2.76 (s, 1H), 
$3.33(\mathrm{~s}, 3 \mathrm{H}), 3.44(\mathrm{~s}, 3 \mathrm{H}), 3.57(\mathrm{dd}, J=4.21,2.38 \mathrm{~Hz}, 1 \mathrm{H}), 3.75(\mathrm{~d}, J=4.21 \mathrm{~Hz}, 1 \mathrm{H})$,

$6.02(\mathrm{~d}, J=2.38 \mathrm{~Hz}, 1 \mathrm{H}) ;{ }^{13} \mathrm{C} \mathrm{NMR}\left(67 \mathrm{MHz}, \mathrm{CDCl}_{3}\right)$ 3.95, 49.7, 50.0, 53.6, 56.9, 67.5, 76.1, 84.2, 96.8, 127.0, 129.1; HRMS: $\left(\mathrm{M}+\mathrm{Na},{ }^{81} \mathrm{Br}\right)$ calcd for $\mathrm{C}_{11} \mathrm{H}_{13} \mathrm{BrO}_{4} \mathrm{Na}, 312.9895$; found 312.9875 .

Alcohol 16. $\operatorname{Red}-\mathrm{Al}^{\circledR}(0.35 \mathrm{ml}, 3.4 \mathrm{M}$ in toluene, $1.19 \mathrm{mmol})$ was added dropwise to a solution of alcohol $15(0.11 \mathrm{~g}, 0.38 \mathrm{mmol})$ in $\mathrm{THF}$ at $-10^{\circ} \mathrm{C}$. After $2 \mathrm{~h}$, the reaction mixture was warmed to $0^{\circ} \mathrm{C}$ and stirred for an additional $1 \mathrm{~h}$. The resulting mixture was poured into saturated $\mathrm{NH}_{4} \mathrm{Cl}$ solution. After extractive workup with ether, the residue was purified by flash chromatography on silica gel 10/90 ethyl acetate/ hexane, followed by 20/80 ethyl acetate/hexane to give alcohol 16 as a white solid (0.101 g, 91\%): mp 113-114 ${ }^{\circ} \mathrm{C}$; IR (film) 3425 (br), 2942, 1643, $967 \mathrm{~cm}^{-1}$; ${ }^{1} \mathrm{H}$ NMR (270 MHz, CDCl $) 1.73$ $(\mathrm{dd}, J=6.53,1.58 \mathrm{~Hz}, 3 \mathrm{H}), 2.73(\mathrm{~s}, 1 \mathrm{H}), 3.30(\mathrm{~s}, 3 \mathrm{H}), 3.41(\mathrm{~s}, 3 \mathrm{H}), 3.42(\mathrm{~d}, J=4.21 \mathrm{~Hz}$, $1 \mathrm{H}), 3.53(\mathrm{dd}, J=4.21,2.57 \mathrm{~Hz}, 1 \mathrm{H}), 5.35(\mathrm{dd}, J=14.75,1.58 \mathrm{~Hz}, 1 \mathrm{H}), 5.92(\mathrm{dq}, J=$ 15.52, 6.53Hz, 1H), $6.03(\mathrm{~d}, J=2.57 \mathrm{~Hz}, 1 \mathrm{H}) ;{ }^{13} \mathrm{C} \mathrm{NMR}\left(67 \mathrm{MHz}, \mathrm{CDCl}_{3}\right) 17.9,50.0$ (intense), 53.5, 56.9, 73.4, 97.2, 127.2, 129.4, 129.7, 131.8; HRMS: $\left(\mathrm{M}+\mathrm{Na},{ }^{81} \mathrm{Br}\right)$ calcd for $\mathrm{C}_{11} \mathrm{H}_{15} \mathrm{BrO}_{4} \mathrm{Na}$, 315.0052; found 315.005.

Ester 11. (Method 2): $n$-BuLi (4.24 $\mathrm{ml}, 2.87 \mathrm{M}$ in hexane, $12.2 \mathrm{mmol}$ ) was added to a solution of alcohol $16(2.361 \mathrm{~g}, 8.1 \mathrm{mmol})$ in ether $(75 \mathrm{~mL})$ at $-78^{\circ} \mathrm{C}$. The reaction was stirred $10 \mathrm{~min}$, then propionic anhydride $(2.10 \mathrm{ml}, 16.4 \mathrm{mmol})$ was added via syringe. The mixture was maintained at $-78^{\circ} \mathrm{C}$ for $15 \mathrm{~min}$, then allowed to warm to rt. After $12 \mathrm{~h}$ the mixture was then poured into saturated $\mathrm{NaHCO}_{3}$ solution and the residue isolated by 
extractive workup with ether. Flash chromatography on silica gel using 10/90 ethyl acetate/hexanes gave ester 11 as a white solid (2.805 g, 100\%).

Ester 36. Diazomethane, generated from N-Methyl-N'-nitro-N-nitrosoguanidine (5.0 g, $3.4 \mathrm{mmol})$ in water $(10 \mathrm{~mL})$ and $2 \mathrm{~N} \mathrm{NaOH}$ solution $(20 \mathrm{~mL})$ was bubbled into a solution of acid $10(1.15 \mathrm{~g}, 3.31 \mathrm{mmol})$ in ether $(100 \mathrm{~mL})$. Upon completion of the reaction (as monitored by TLC), a few drops of $\mathrm{AcOH}$ was added and the reaction mixture concentrated in vacuo. The residue was purified by flash chromatography on Florisil with 20/80 ethyl acetate/hexane to give methyl ester 36 as a yellow oil $(0.88 \mathrm{~g}, 73 \%)$ : IR (film) 2969, $1736 \mathrm{~cm}_{-}{ }^{1}$; ${ }^{1} \mathrm{H}$ NMR (270 MHz, $\left.\mathrm{CDCl}_{3}\right) 1.07$ (d, $\left.J=6.53 \mathrm{~Hz}, 3 \mathrm{H}\right), 1.13(\mathrm{~d}, J$ $=7.13 \mathrm{~Hz}, 3 \mathrm{H}), 2.42(\mathrm{dq}, J=8.58,7.13 \mathrm{~Hz}, 1 \mathrm{H}), 3.04(\mathrm{dqd}, J=8.58,6.73,2.38 \mathrm{~Hz}, 1 \mathrm{H})$, 3.30 (s, 3H), 3.45 (s, 3H), $3.61(\mathrm{dd}, J=4.16,2.38 \mathrm{~Hz}, 1 \mathrm{H}), 3.68(\mathrm{~s}, 3 \mathrm{H}), 4.00(\mathrm{~d}, J=4.16$ $\mathrm{Hz}, 1 \mathrm{H}), 6.10(\mathrm{~d}, J=2.38 \mathrm{~Hz}, 1 \mathrm{H}), 6.21(\mathrm{~d}, J=10.89 \mathrm{~Hz}, 1 \mathrm{H}) ;{ }^{13} \mathrm{C} \mathrm{NMR}(67 \mathrm{MHz}$, $\left.\mathrm{CDCl}_{3}\right) 15.5,19.2,35.8,45.6,49.3,49.6,49.6,51.8,54.2,97.2,124.3,126.9,128.0$, $145.5,175.8$.

Diol 37. A mixture of $\mathrm{OsO}_{4}(0.13 \mathrm{~g}, 4 \%$ wt. in water, $0.51 \mathrm{mmol})$, ester 36 (0.754 g, 2.09 mmol), NMO (0.734 g, $6.27 \mathrm{mmol})$ and methanesulfonamide $(0.397 \mathrm{~g}, 4.17 \mathrm{mmol})$ in acetone $(16 \mathrm{~mL})$ and water $(2 \mathrm{~mL})$ was stirred for $48 \mathrm{~h}$. The reaction mixture was quenched with $\mathrm{Na}_{2} \mathrm{SO}_{3}$ and allowed to stir for 30 min. After extractive workup with ethyl acetate, the residue was purified by flash chromatography over Florisil with 10/90 ethyl acetate/hexane followed by 20/80 ethyl acetate/hexane to give diol $\mathbf{3 7}$ as a white solid (0.20 g, $31 \%$ brsm); mp $118-120{ }^{\circ} \mathrm{C}$; IR (film) 3467 (br), 2944, $1733 \mathrm{~cm}^{-} ;{ }^{1} \mathrm{H}$ NMR $\left(270 \mathrm{MHz}, \mathrm{CDCl}_{3}\right) 0.98$ (d, $\left.J=6.93 \mathrm{~Hz}, 3 \mathrm{H}\right), 1.18(\mathrm{~d}, J=7.13 \mathrm{~Hz}, 3 \mathrm{H}), 1.87(\mathrm{~m}, 1 \mathrm{H})$, 
$2.82(\mathrm{~m}, 1 \mathrm{H}), 3.32(\mathrm{~s}, 3 \mathrm{H}), 3.43(\mathrm{~s}, 3 \mathrm{H}),(\mathrm{dd}, J=3.96,218 \mathrm{~Hz}, 1 \mathrm{H}), 3.68(\mathrm{~s}, 3 \mathrm{H}), 4.05($ $\mathrm{d}, J=2.18 \mathrm{~Hz}, 1 \mathrm{H}), 4.28(\mathrm{~s}, 1 \mathrm{H}), 6.23(\mathrm{~d}, J=2.18 \mathrm{~Hz}, 1 \mathrm{H}) ;{ }^{13} \mathrm{C} \mathrm{NMR}\left(67 \mathrm{MHz}, \mathrm{CDCl}_{3}\right)$ 8.5, 15.5, 36.4, 45.9, 49.3, 50.0, 50.8, 52.0, 53.0, 73.4, 79.2, 127.3, 132.1, 177.3; HRMS: $\left(\mathrm{M}+\mathrm{Na},{ }^{81} \mathrm{Br}\right.$ ) calcd for $\mathrm{C}_{15} \mathrm{H}_{23} \mathrm{BrO}_{7} \mathrm{Na}, 419.0525$; found 419.051 .

Epoxyalcohol 38. A mixture of $\mathrm{OsO}_{4}(0.04 \mathrm{~g}, 4 \%$ wt. in water, $0.15 \mathrm{mmol})$, methyl ester $36(0.225 \mathrm{~g}, 0.62 \mathrm{mmol}), \mathrm{K}_{3} \mathrm{Fe}(\mathrm{CN})_{6}(1.23 \mathrm{~g}, 3.74 \mathrm{mmol})$, methanesulfonamide $(0.119 \mathrm{~g}$, $1.25 \mathrm{mmol})$ and $\mathrm{K}_{2} \mathrm{CO}_{3}(0.517 \mathrm{~g}, 3.74 \mathrm{mmol})$ in $t-\mathrm{BuOH}(5 \mathrm{~mL})$ and water $(5 \mathrm{~mL})$ was stirred for $48 \mathrm{~h}$. The mixture was quenched with $\mathrm{Na}_{2} \mathrm{SO}_{3}$ and allowed to stir for $30 \mathrm{~min}$. After extractive workup with ethyl acetate, the residue was purified by flash chromatography over Florisil with 10/90 ethyl acetate/hexane, followed by 20/80 ethyl acetate/hexane to give diol 37 as a white solid $(0.150 \mathrm{~g}, 66 \%) ; \mathrm{mp} 60-61{ }^{\circ} \mathrm{C}$; IR (film) 3471 (br), 2945, $1782 \mathrm{~cm}^{-1}$; ${ }^{1} \mathrm{H}$ NMR (270 MHz, $\left.\mathrm{CDCl}_{3}\right) 0.90$ (d, $\left.J=6.93 \mathrm{~Hz}, 3 \mathrm{H}\right), 1.13$ (d, $J=6.73 \mathrm{~Hz}, 3 \mathrm{H}), 2.87(\mathrm{~m}, 2 \mathrm{H}), 2.93(\mathrm{~d}, J=8.91 \mathrm{~Hz}, 1 \mathrm{H}), 3.31(\mathrm{~s}, 3 \mathrm{H}), 3.34(\mathrm{~s}, 3 \mathrm{H})$, $3.75(\mathrm{~d}, J=0.99 \mathrm{~Hz}, 1 \mathrm{H}), 4.07(\mathrm{dd}, J=8.91,0.99 \mathrm{~Hz}, 1 \mathrm{H}), 4.95(\mathrm{~d}, J=3.96 \mathrm{~Hz}, 1 \mathrm{H})$, 6.30 (s, 1H); 13C NMR (67 MHz, $\left.\mathrm{CDCl}_{3}\right)$ 8.93, 9.62, 36.42, 41.05, 49.24, 51.25, 57.16, 61.59, 70.26, 77.50, 97.76, 123.15, 136.10, 177.39; HRMS: $\left(\mathrm{M}+\mathrm{Na},{ }^{81} \mathrm{Br}\right)$ calcd for $\mathrm{C}_{14} \mathrm{H}_{19} \mathrm{BrO}_{6} \mathrm{Na}, 387.0263$; found 387.0262 .

Epoxyketone 39. DMSO $(0.137 \mathrm{~mL}, 1.93 \mathrm{mmol})$ was added dropwise to a solution of oxalyl chloride ( $0.072 \mathrm{~mL}, 0.83 \mathrm{mmol})$ in $\mathrm{CH}_{2} \mathrm{Cl}_{2}(3 \mathrm{~mL})$ at $-78{ }^{\circ} \mathrm{C}$. After $5 \mathrm{~min}$, epoxy alcohol $38(0.100 \mathrm{~g}, 0.28 \mathrm{mmol})$ in $\mathrm{CH}_{2} \mathrm{Cl}_{2}(3 \mathrm{~mL})$ was added to the reaction mixture. After $10 \mathrm{~min}, \mathrm{NEt}_{3}(0.349 \mathrm{~mL}, 2.48 \mathrm{mmol})$ was added and the mixture allowed to warm 
to rt. After $2 \mathrm{~h}$, the mixture was isolated via extractive workup with ether and the residue purified by flash chromatography on silica gel with 10/90 ethyl acetate/hexane, followed by $20 / 80$ ethyl acetate/hexane to give epoxyketone 39 as a yellow oil $(0.099 \mathrm{~g}, 100 \%)$ : IR (film) 2976, $1785 \mathrm{~cm}^{-1} ;{ }^{1} \mathrm{H}-\mathrm{NMR}\left(270 \mathrm{MHz}, \mathrm{CDCl}_{3}\right) 0.85(\mathrm{~d}, J=7.13 \mathrm{~Hz}, 3 \mathrm{H}), 1.16$ (d, $J$ $=7.13 \mathrm{~Hz}, 3 \mathrm{H}), 2.92(\mathrm{~m}, 1 \mathrm{H}), 3.02(\mathrm{~m}, 1 \mathrm{H}), 3.33(\mathrm{~s}, 3 \mathrm{H}), 3.34(\mathrm{~s}, 3 \mathrm{H}), 3.87(\mathrm{~s}, 1 \mathrm{H}), 4.99$ $(\mathrm{d}, J=4.75 \mathrm{~Hz}, 1 \mathrm{H}), 6.50(\mathrm{~s}, 1 \mathrm{H}) ;{ }_{13} \mathrm{C} \mathrm{NMR}\left(67 \mathrm{MHz}, \mathrm{CDCl}_{3}\right)$ 9.3, 9.6, 36.5, 40.5, 50.6, 51.4, 56.4, 62.0, 76.6, 95.2, 123.0, 135.3, 176.7, 196.0; HRMS: $\left(\mathrm{MH}^{+}\right)$calcd for $\mathrm{C}_{14} \mathrm{H}_{18} \mathrm{BrO}_{6}, 361.0287$; found 361.0308 .

o-Quinone Monoketal 9. A solution of 1,2-diiodoethane $(0.437 \mathrm{~g}, 1.55 \mathrm{mmol})$ in THF $(10 \mathrm{~mL})$ was added to a mixture of Sm powder $(0.236 \mathrm{~g}, 1.56 \mathrm{mmol})$ in THF $(10 \mathrm{~mL})$ at rt. After $1.5 \mathrm{~h}$, the reaction mixture was cooled to $0^{\circ} \mathrm{C}$ and epoxyketone $39(0.070 \mathrm{~g}, 0.19$ mmol) in a (1:2) methanol/THF mixture was added. The reaction mixture was allowed to slowly warm to rt. After $24 \mathrm{~h}$, the mixture was washed with saturated $\mathrm{K}_{2} \mathrm{CO}_{3}$ solution. After extractive workup with ether, the residue was purified by flash chromatography over silica gel with 10/90 ethyl acetate/hexane to give $o$-quinone monoketal 9 as a yellow oil (0.039g, 59\%): IR (film) 2923, $1785 \mathrm{~cm}^{-1} ;{ }^{1}$-H NMR (270 MHz, $\left.\mathrm{CDCl}_{3}\right) 0.75$ (d, $J=$ $7.32 \mathrm{~Hz}, 3 \mathrm{H}), 1.18(\mathrm{~d}, J=7.32 \mathrm{~Hz}, 3 \mathrm{H}), 2.98(\mathrm{~m}, 1 \mathrm{H}), 3.17(\mathrm{~m}, 1 \mathrm{H}), 3.35(\mathrm{~s}, 3 \mathrm{H}), 3.37$ (s, $3 \mathrm{H}), 5.24(\mathrm{dd}, J=5.15,1.58 \mathrm{~Hz}, 1 \mathrm{H}), 6.23(\mathrm{~d}, J=1.58 \mathrm{~Hz}, 1 \mathrm{H}), 6.78(\mathrm{~s}, 1 \mathrm{H}) ;{ }^{13} \mathrm{C} \mathrm{NMR}$ (67 MHz, $\left.\mathrm{CDCl}_{3}\right)$ 9.87, 9.94, 38.0, 40.5, 50.4, 80.0, 92.9, 118.5, 123.4, 137.6, 146.3, 177.0, 192.2; HRMS: (M+Na) calcd for $\mathrm{C}_{14} \mathrm{H}_{17} \mathrm{BrO}_{5} \mathrm{Na}, 367.0157$; found 367.0178. 


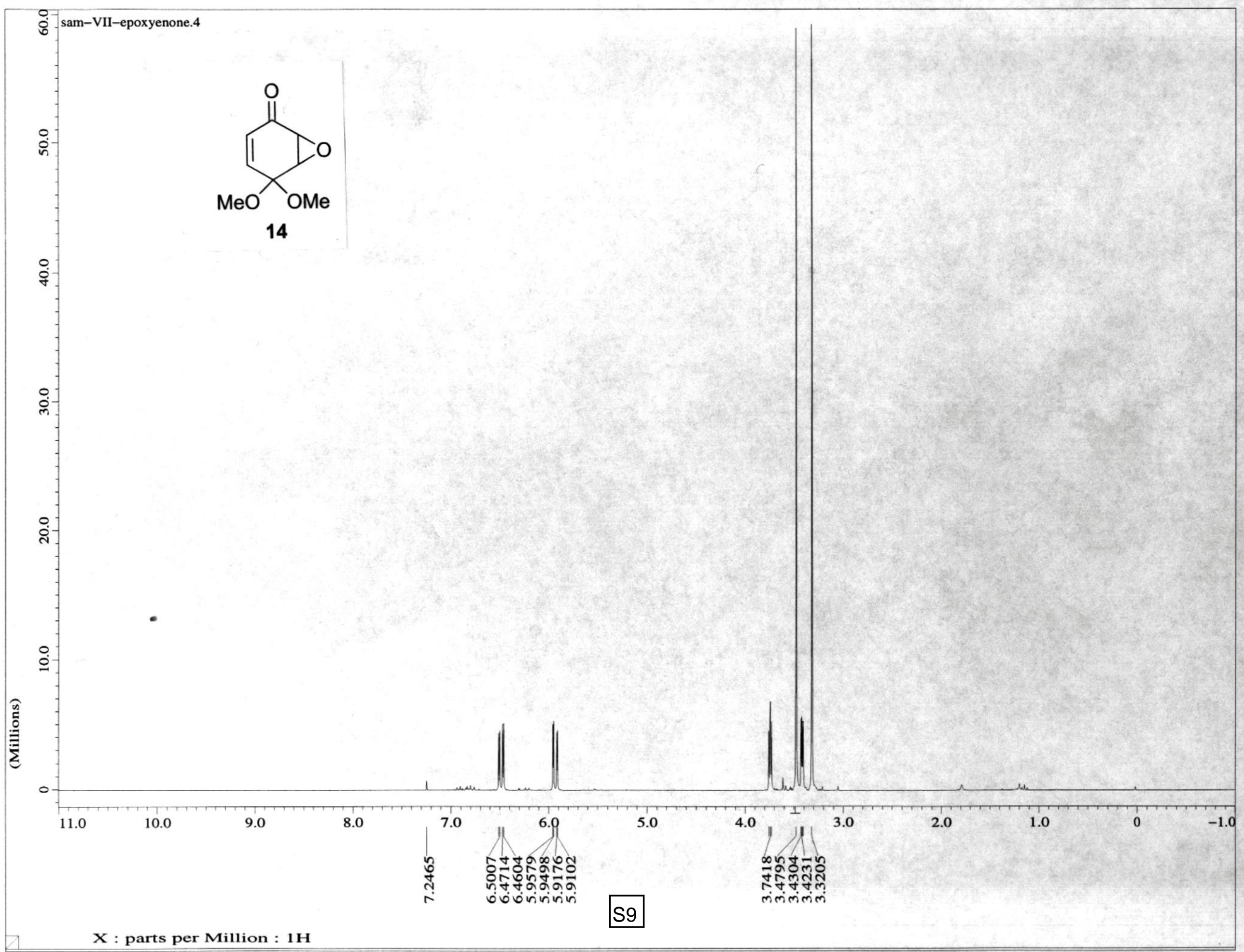




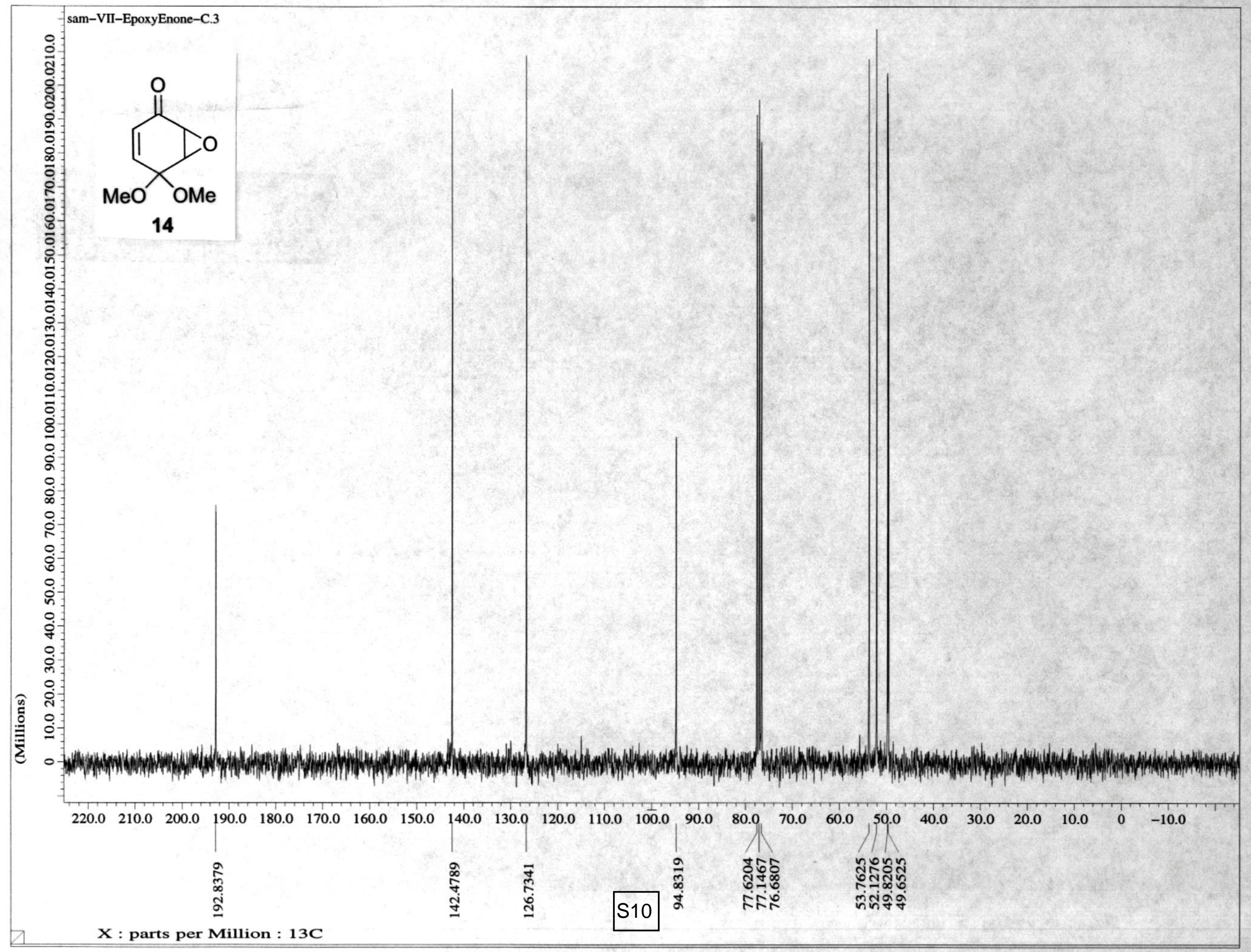




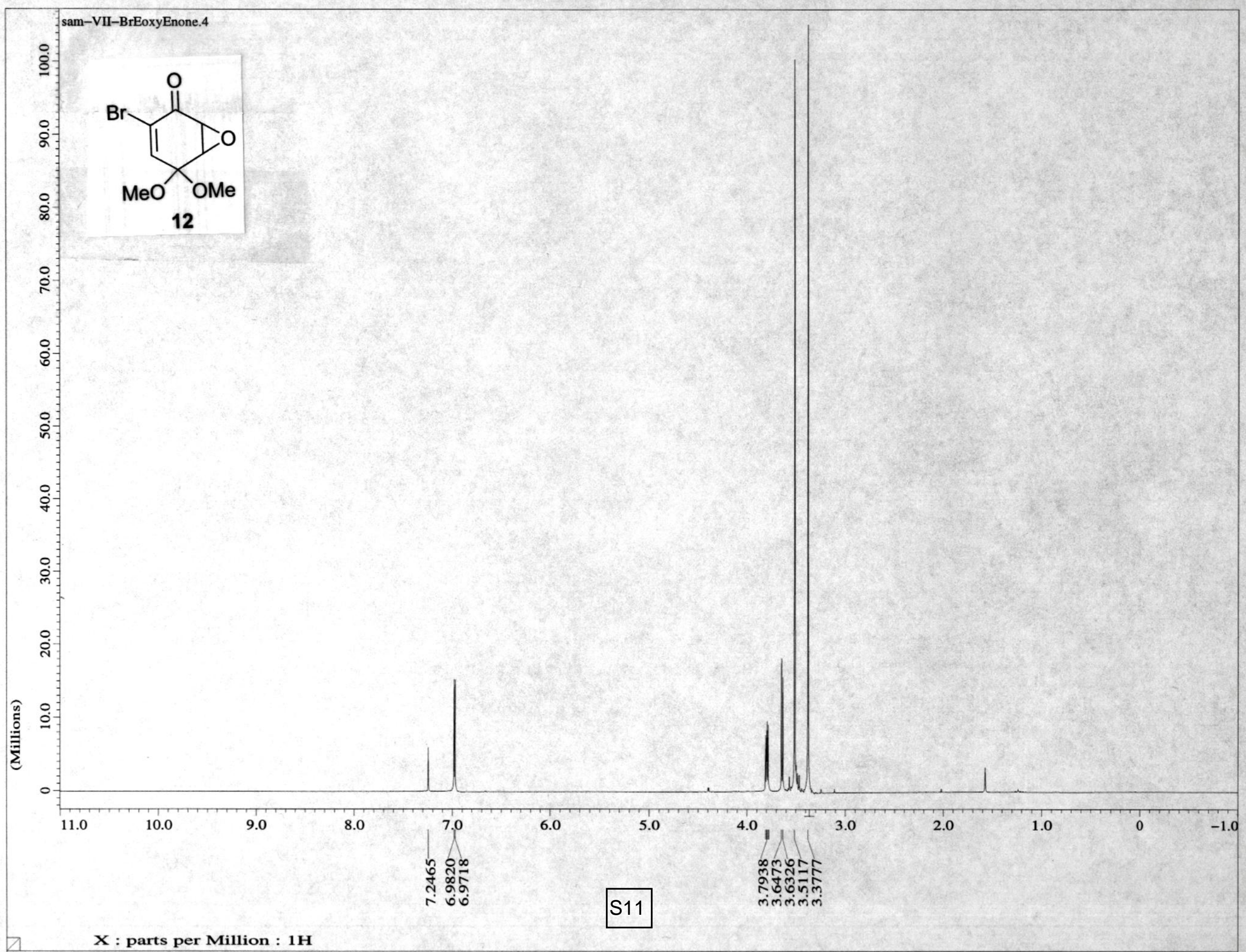




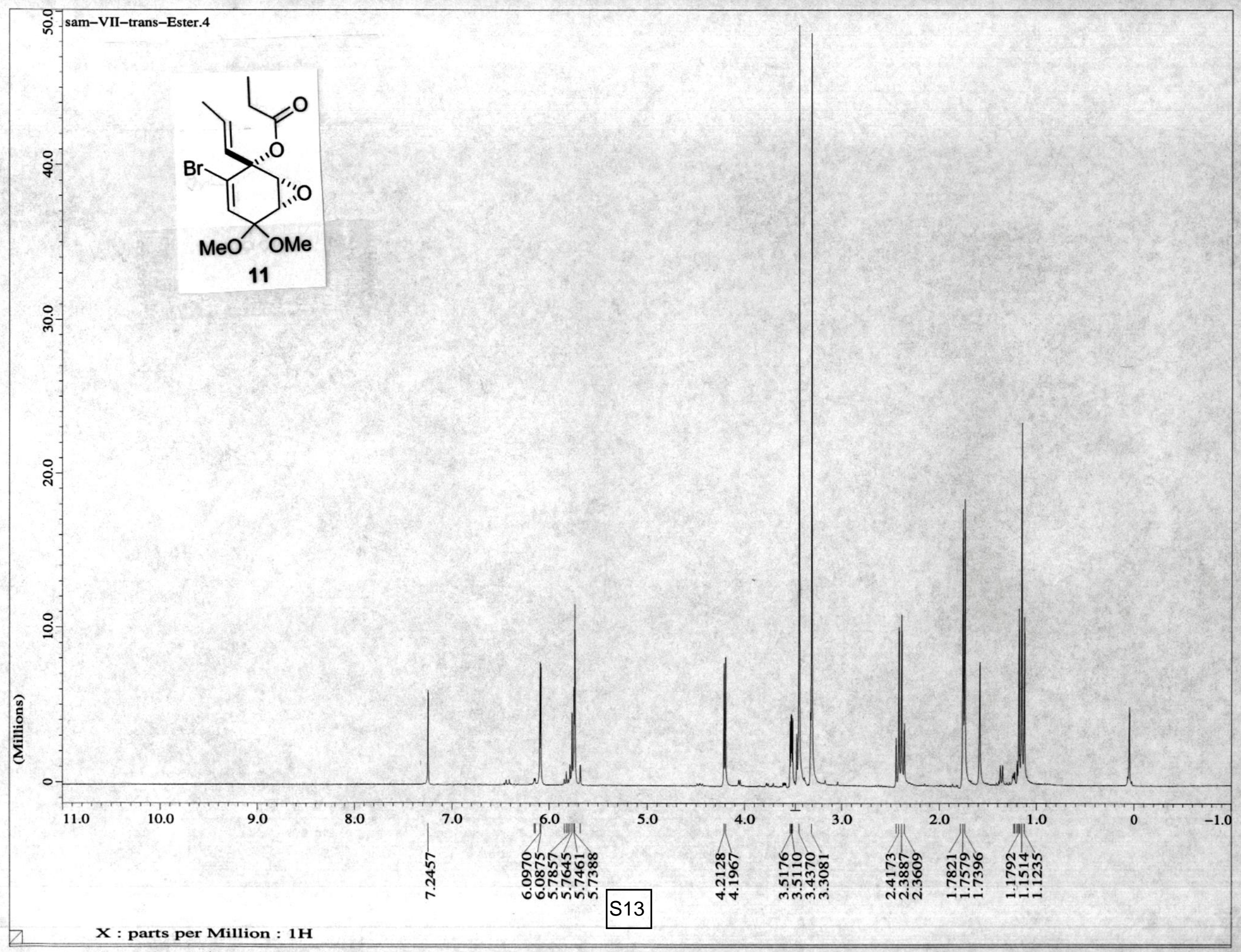




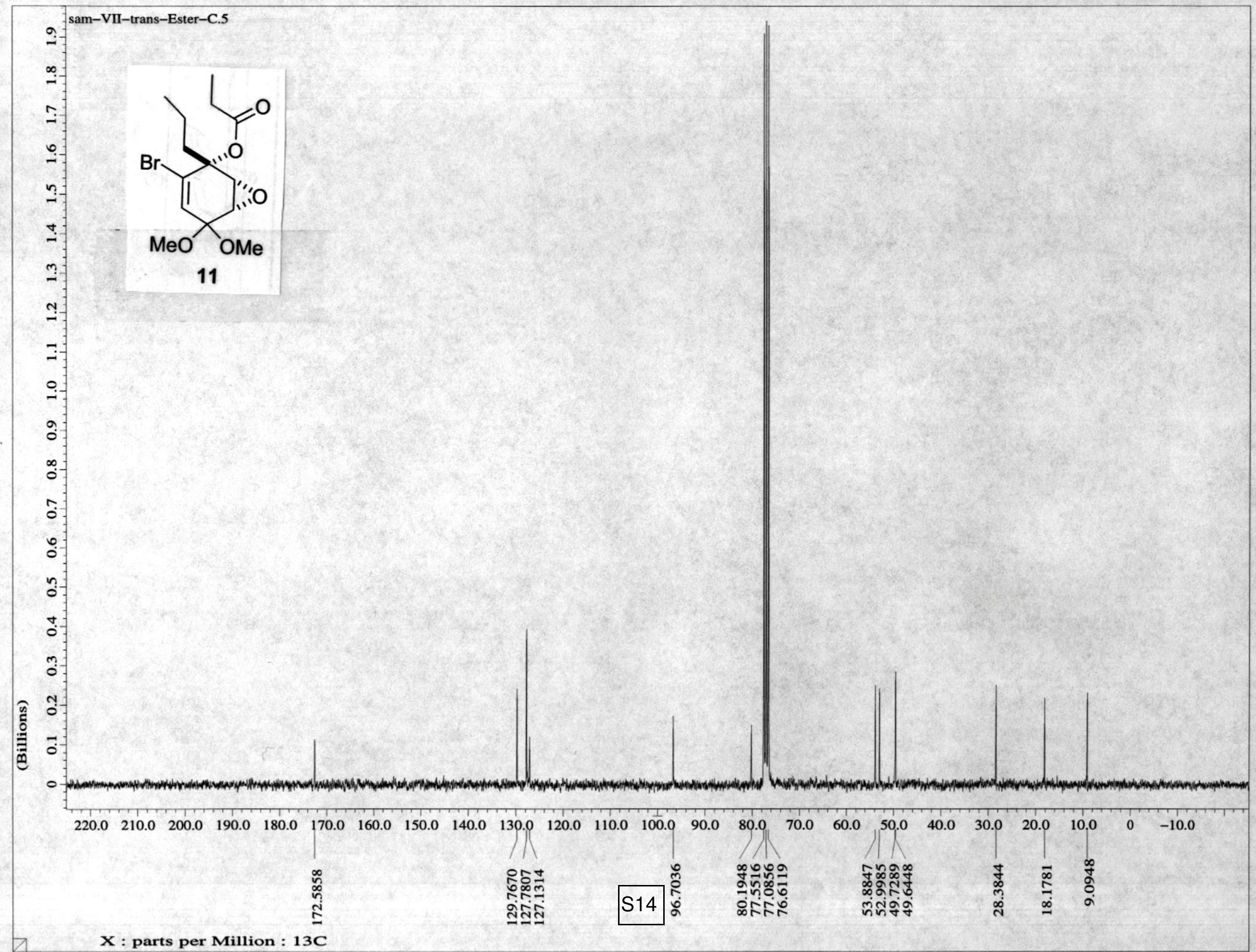




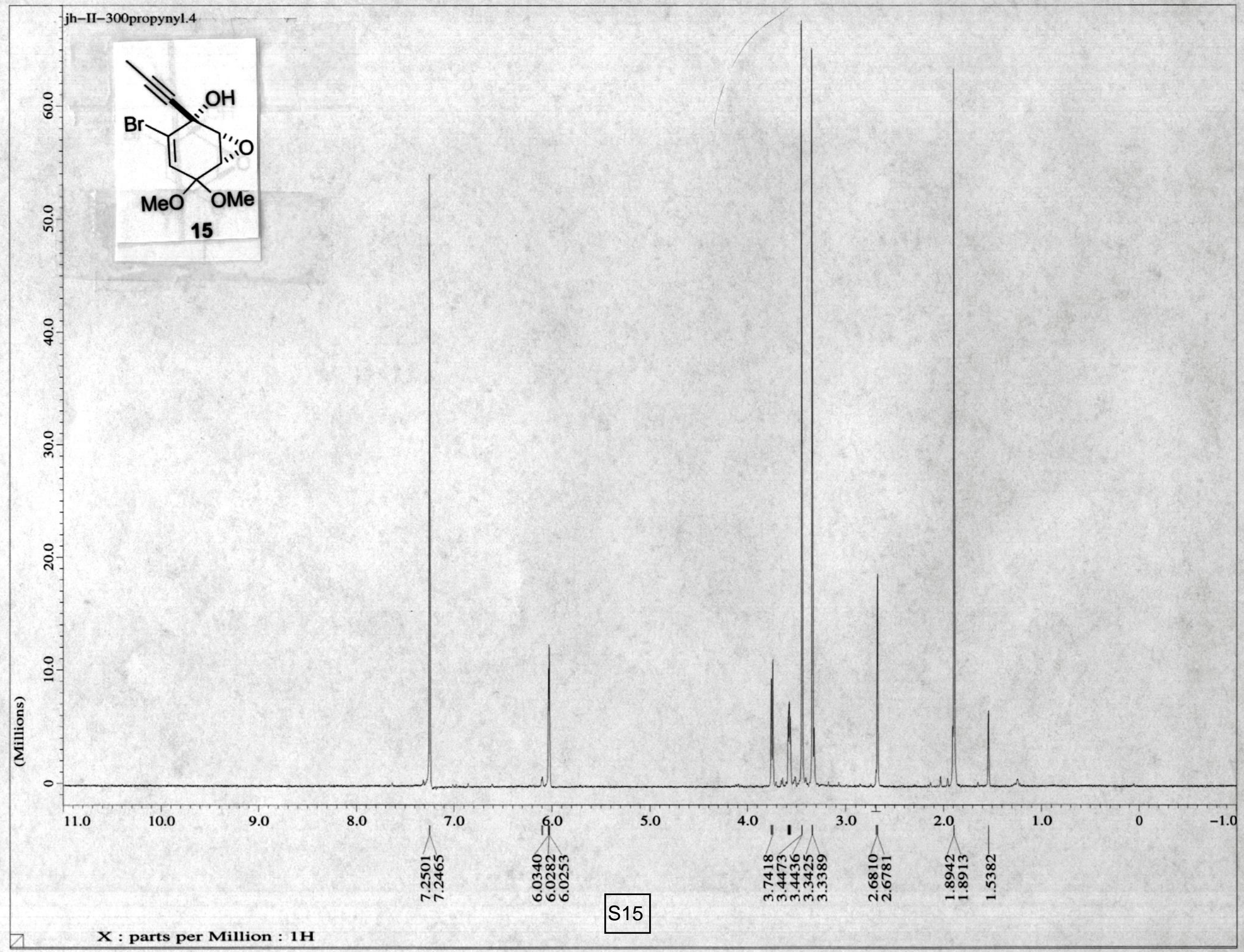




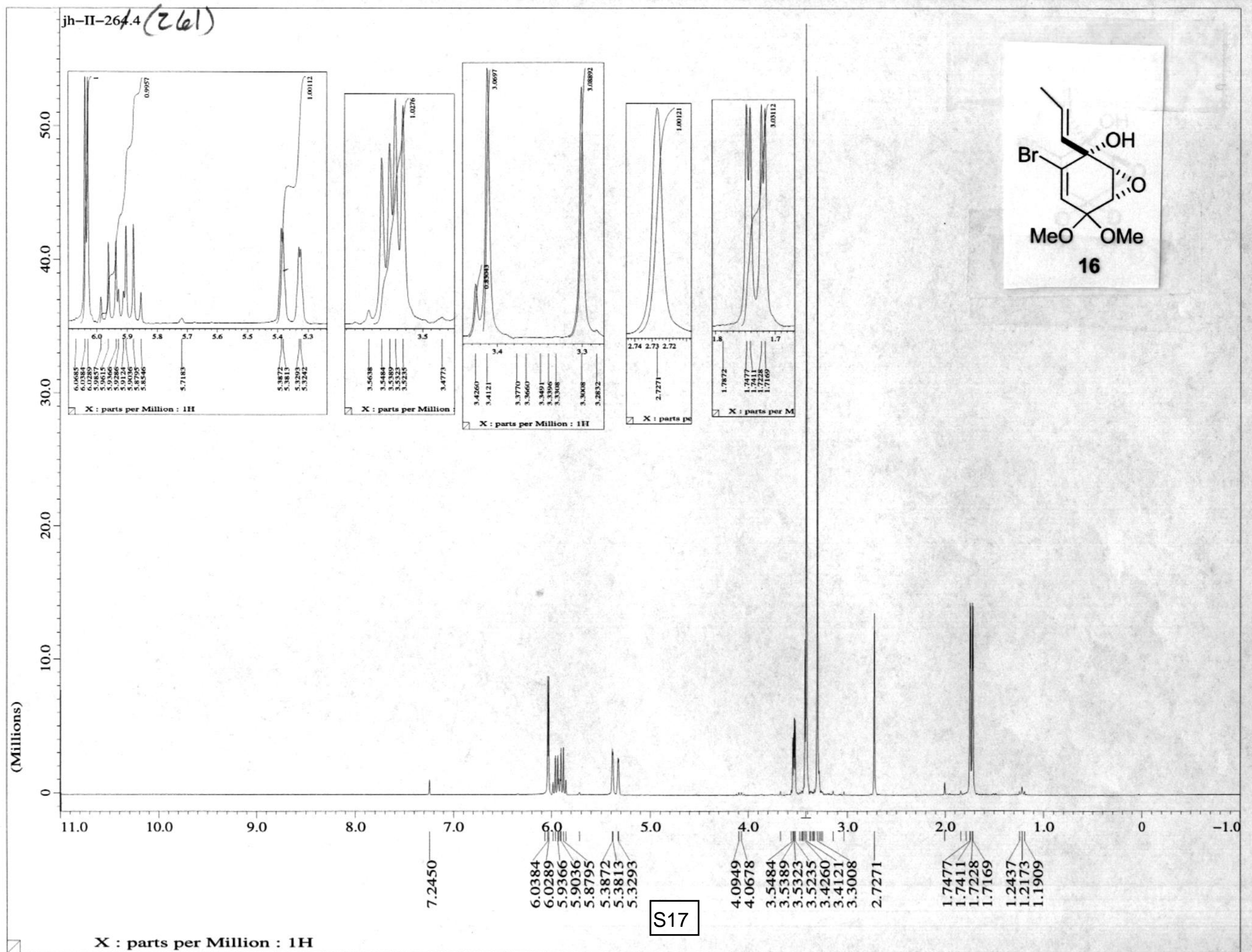




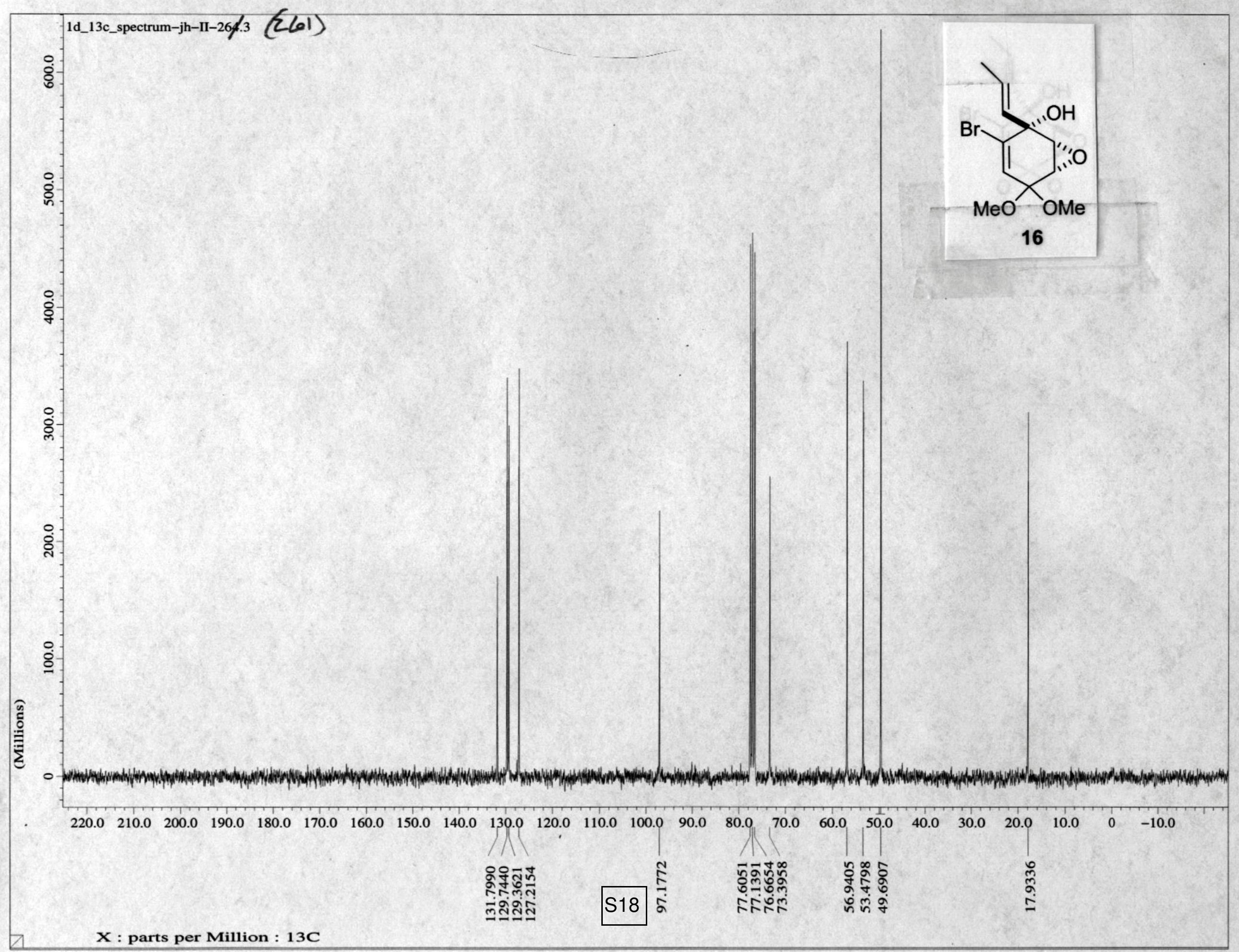




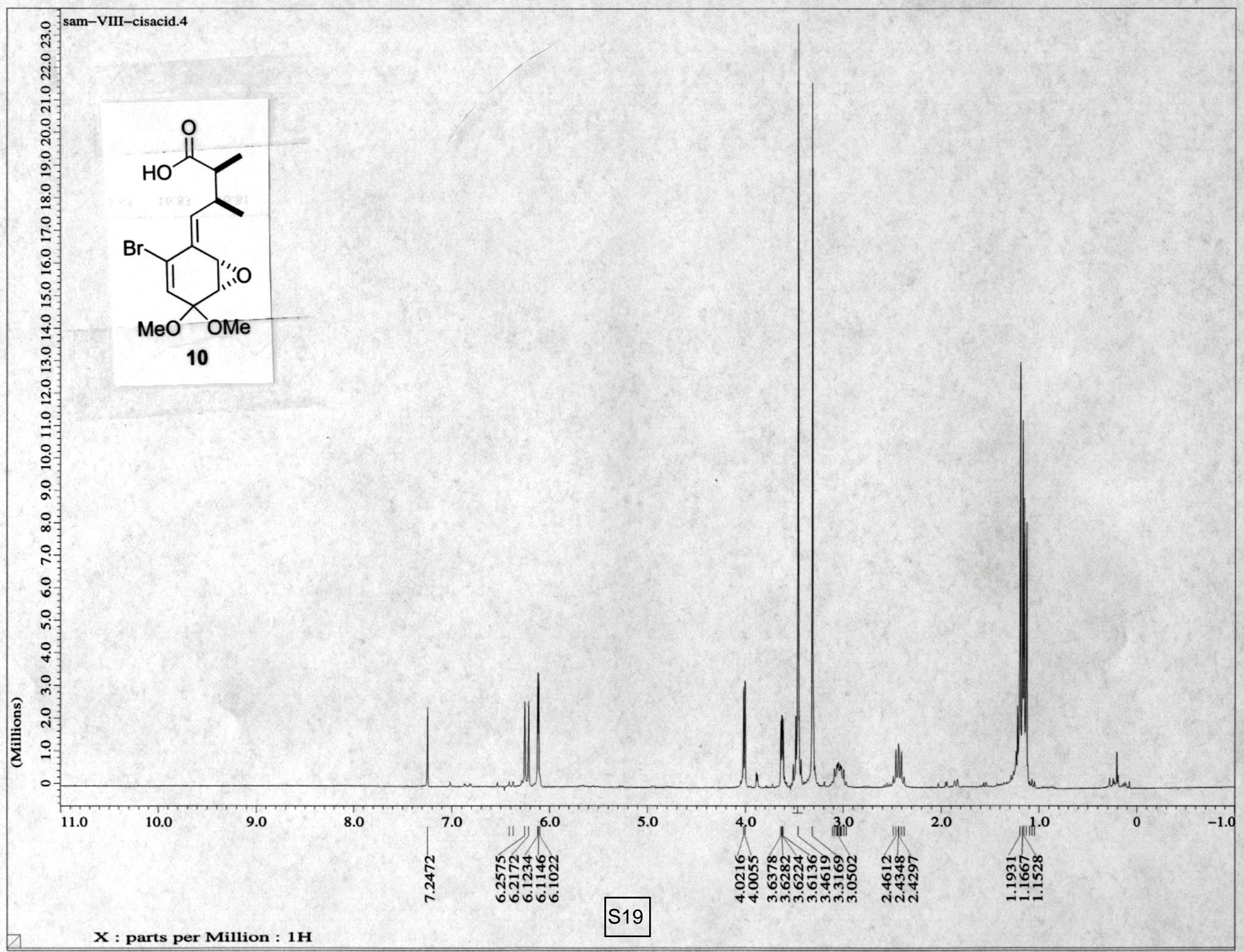




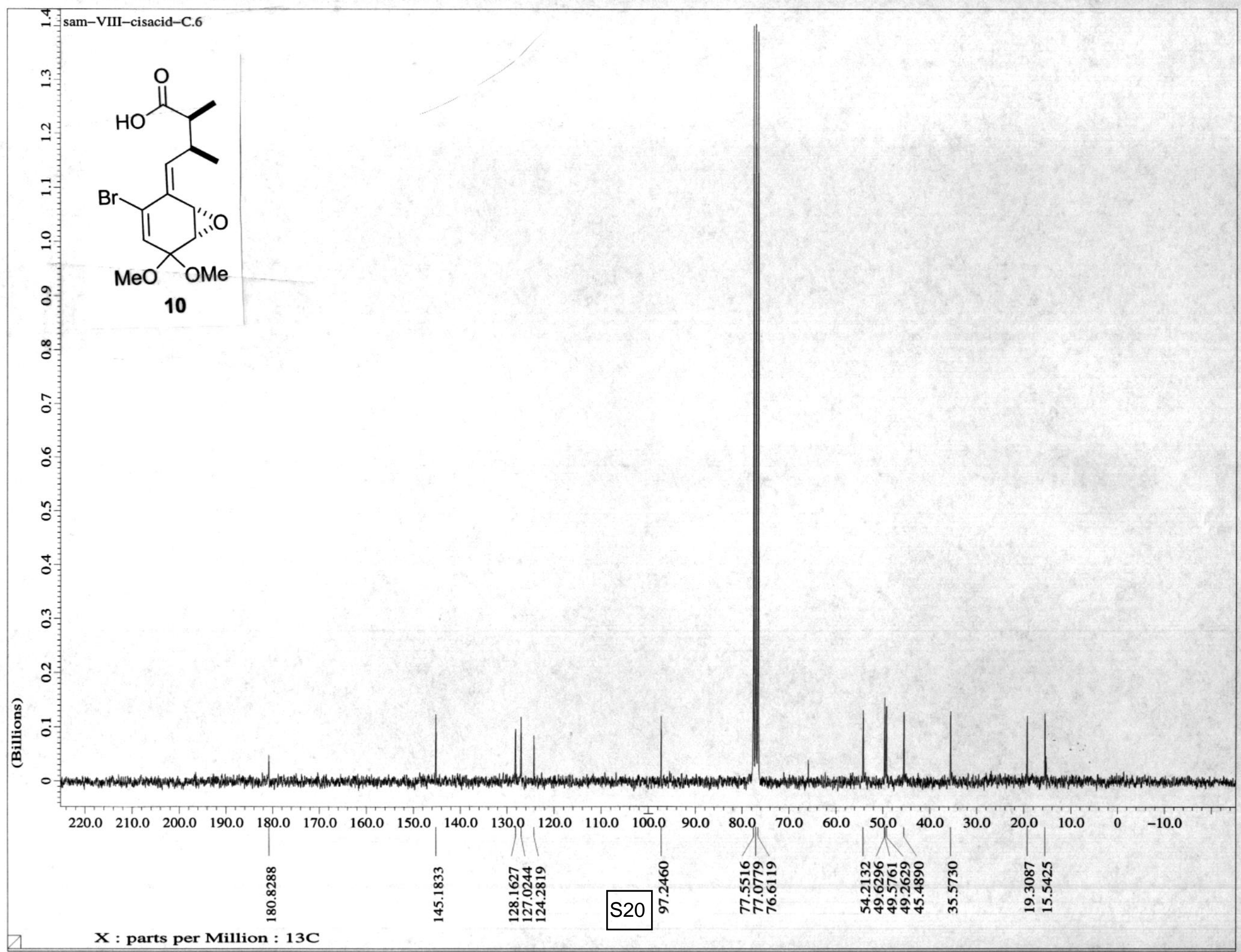




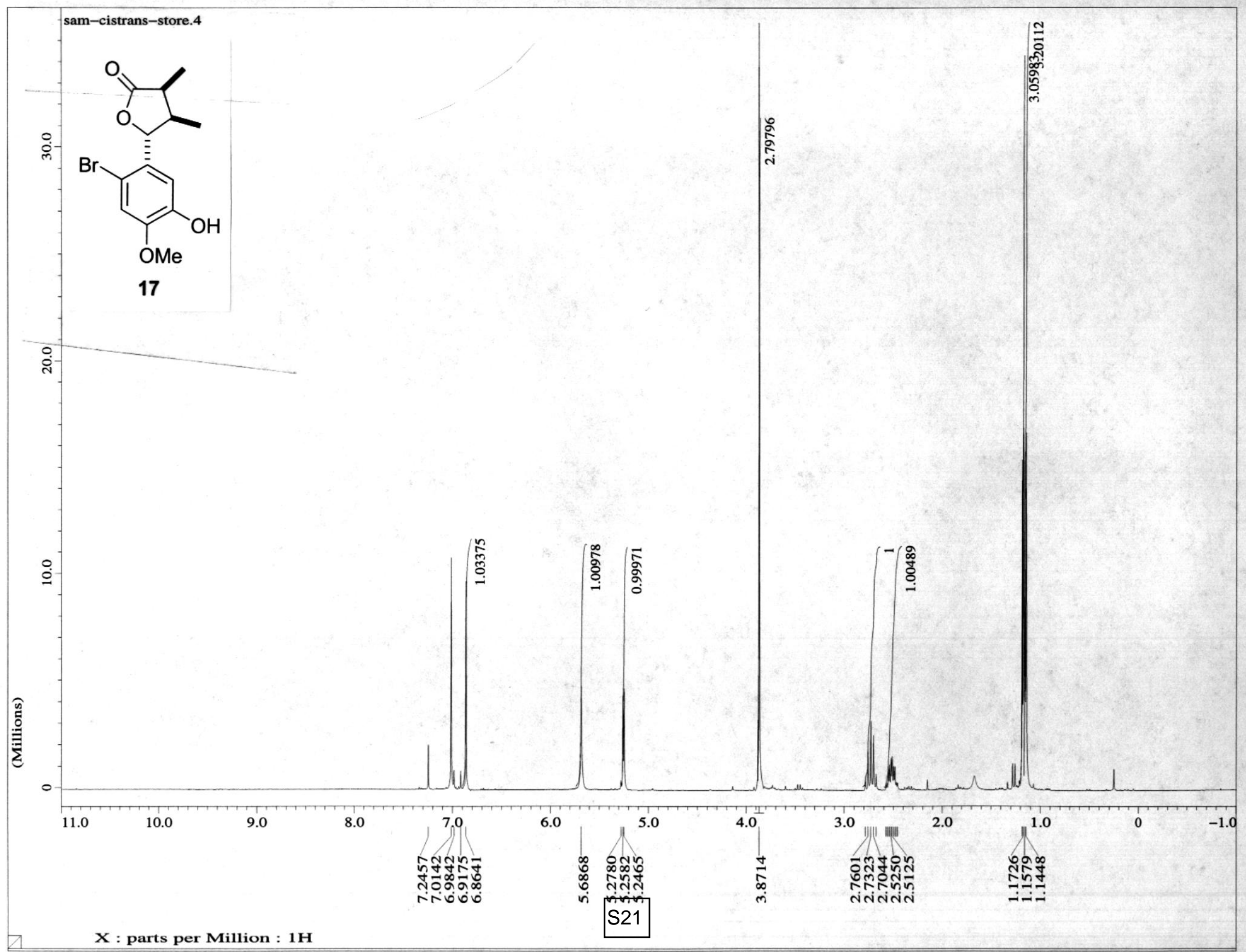




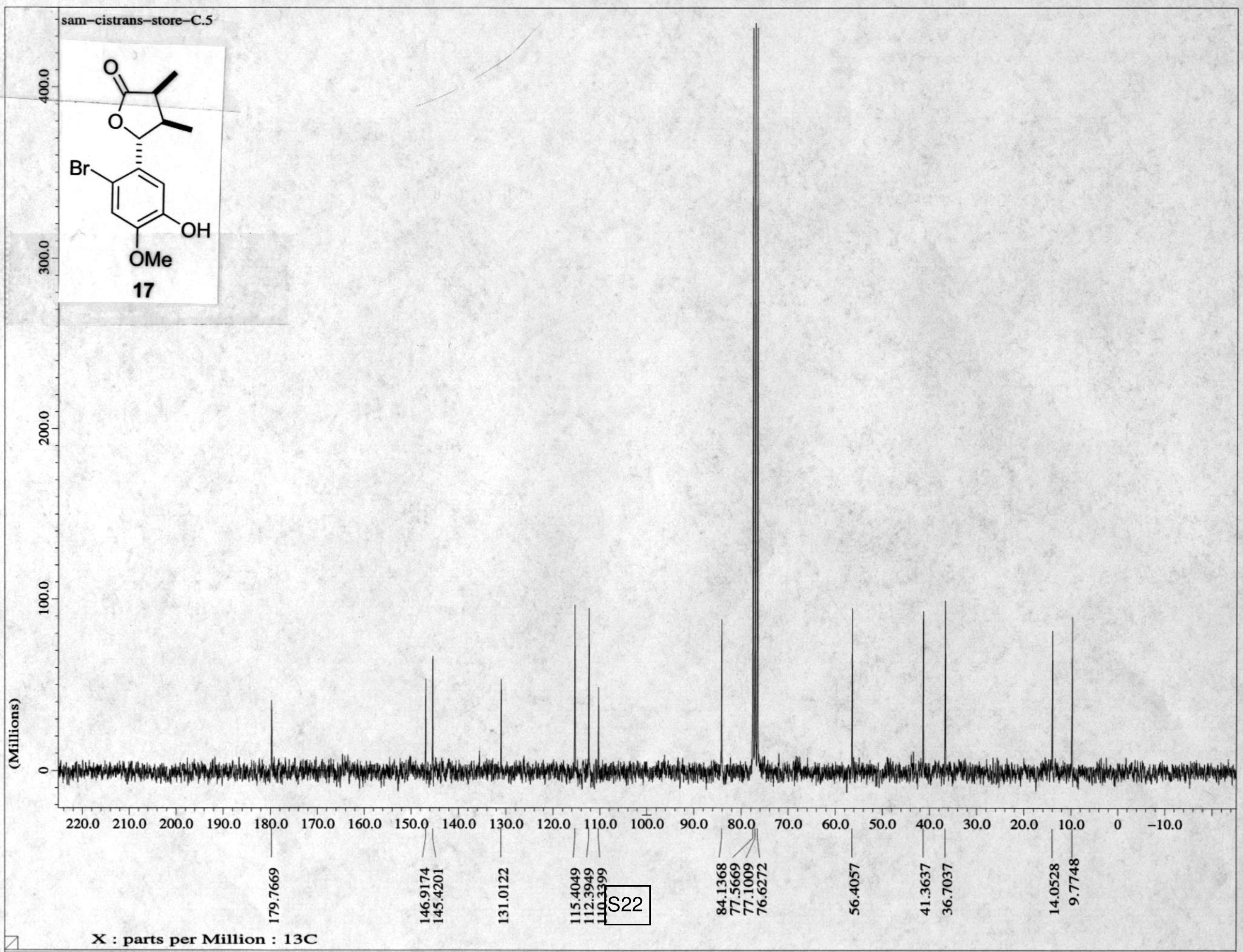




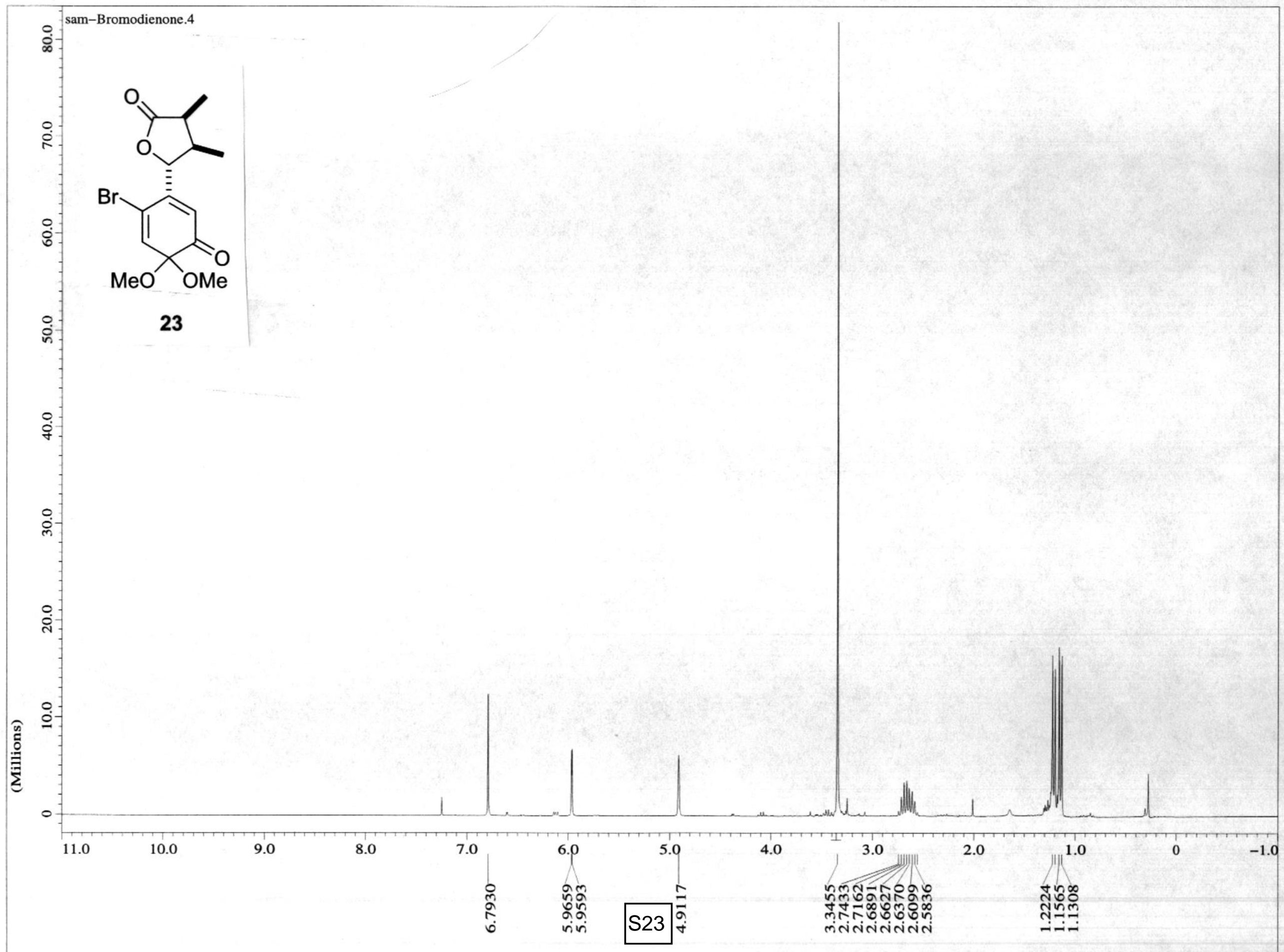




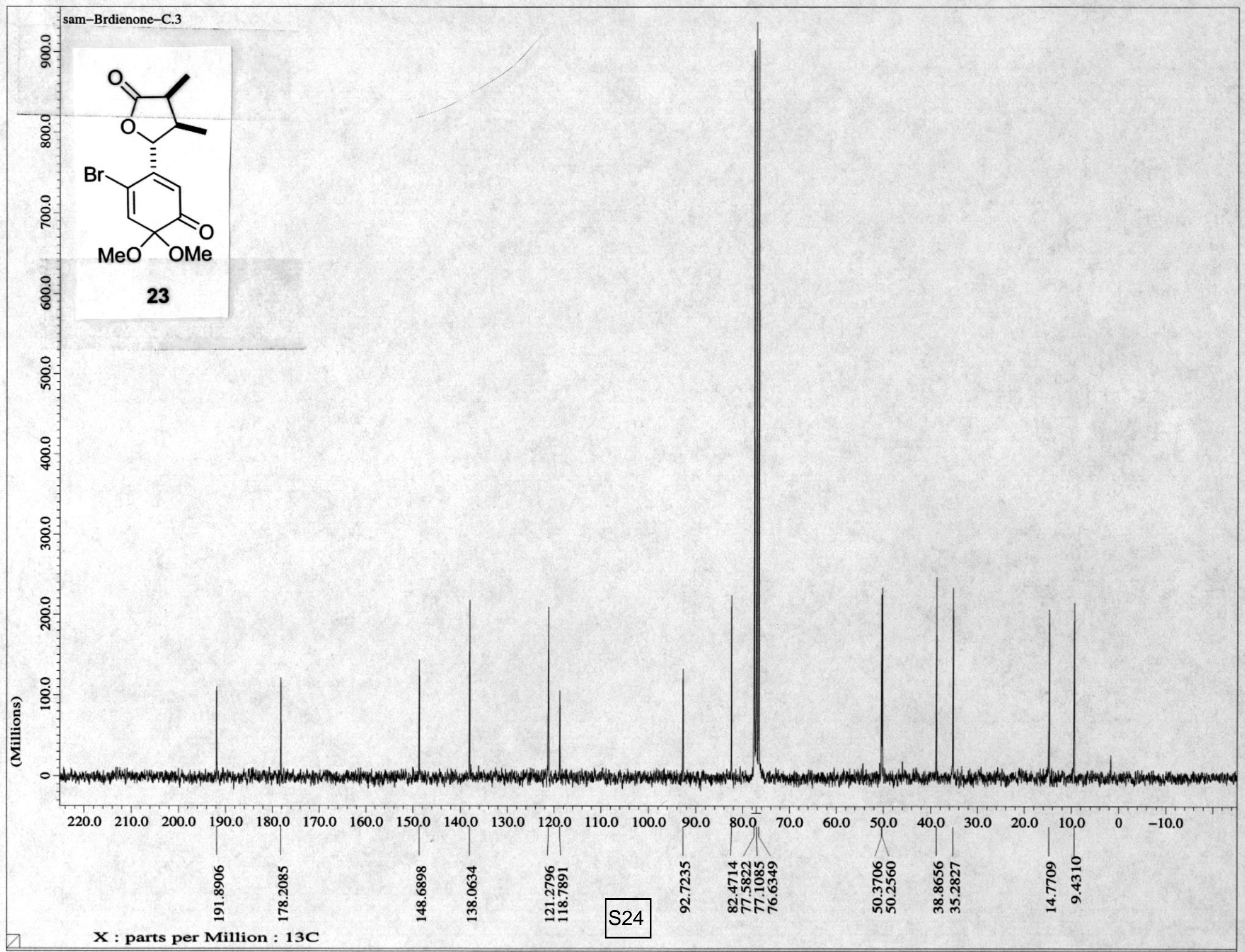




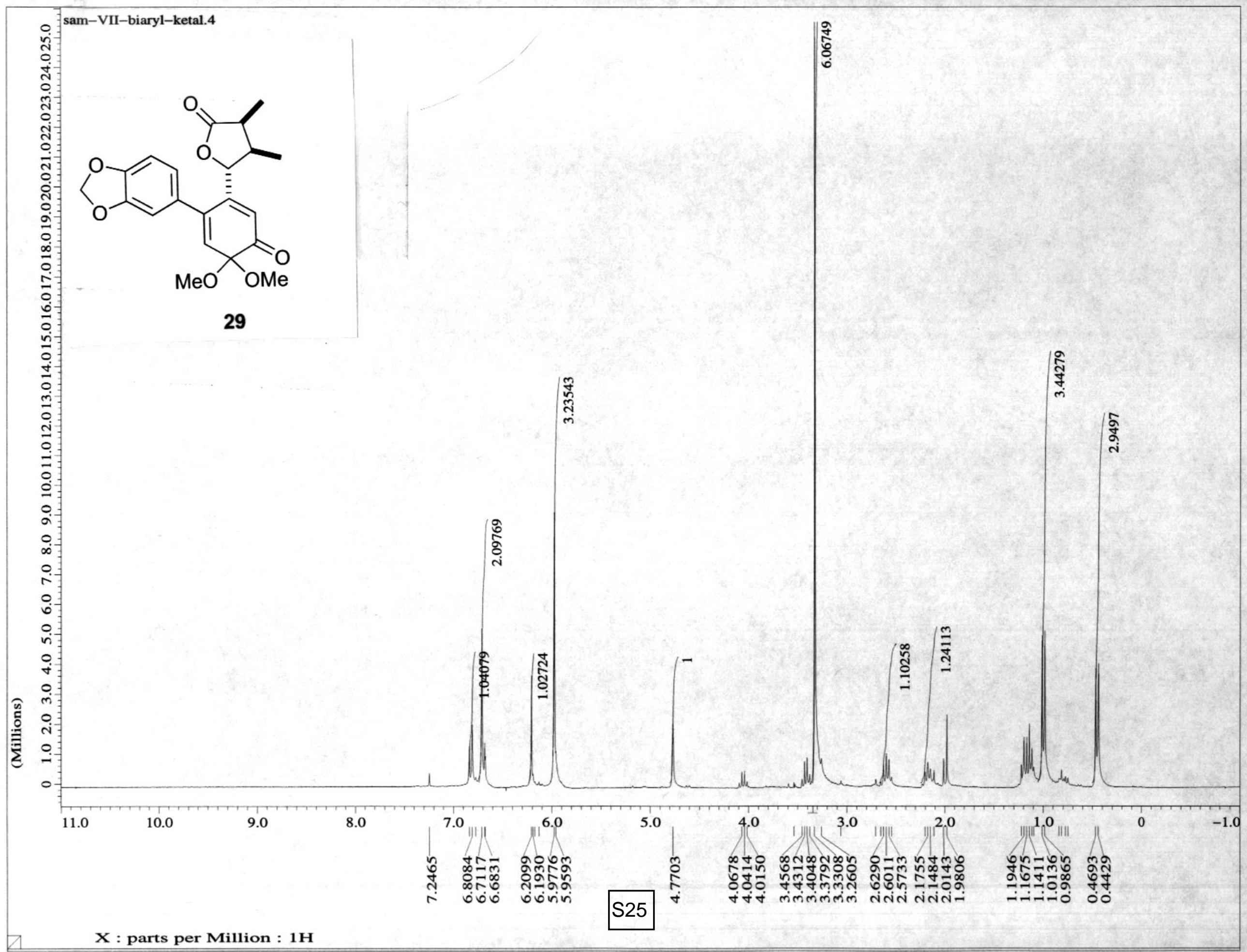




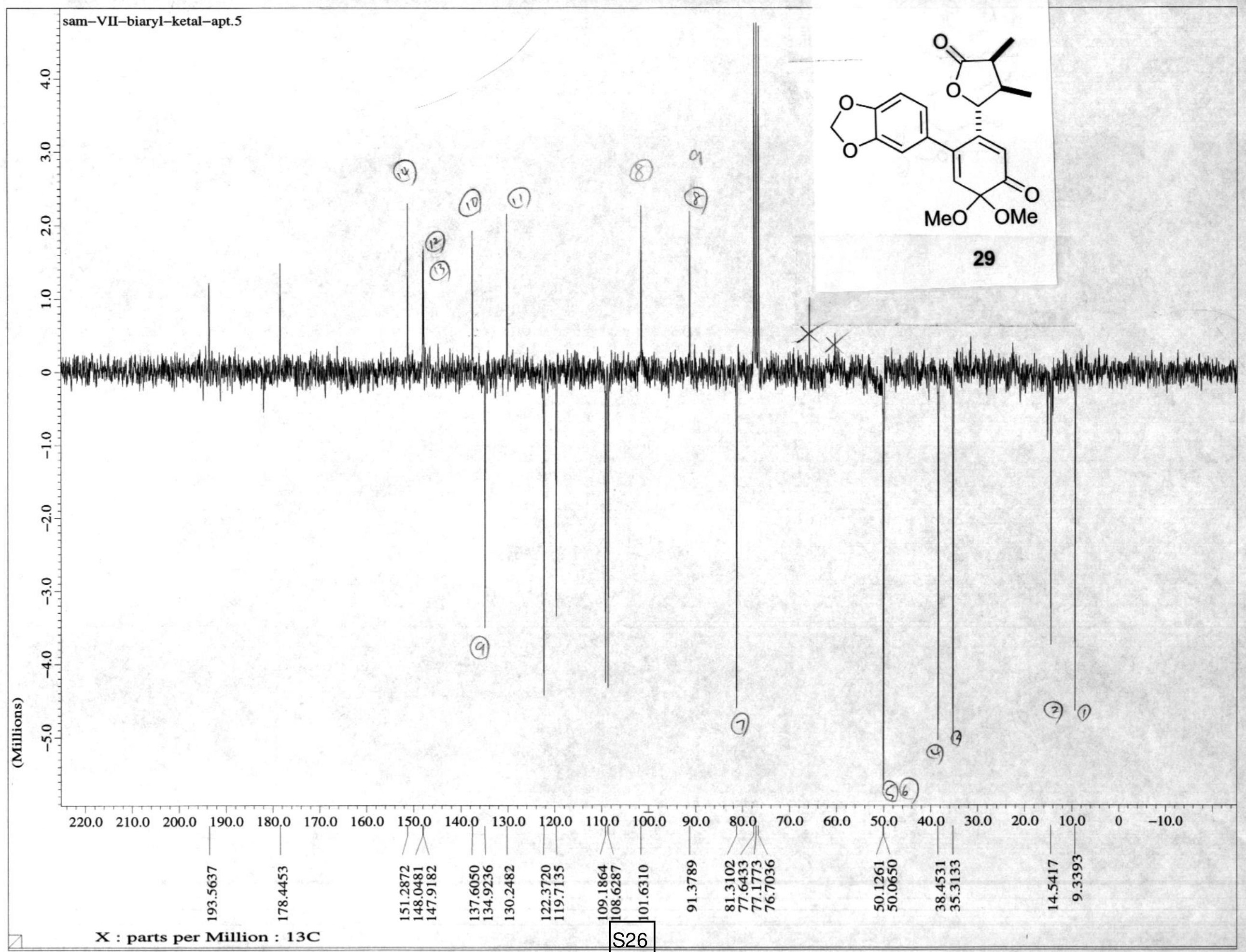




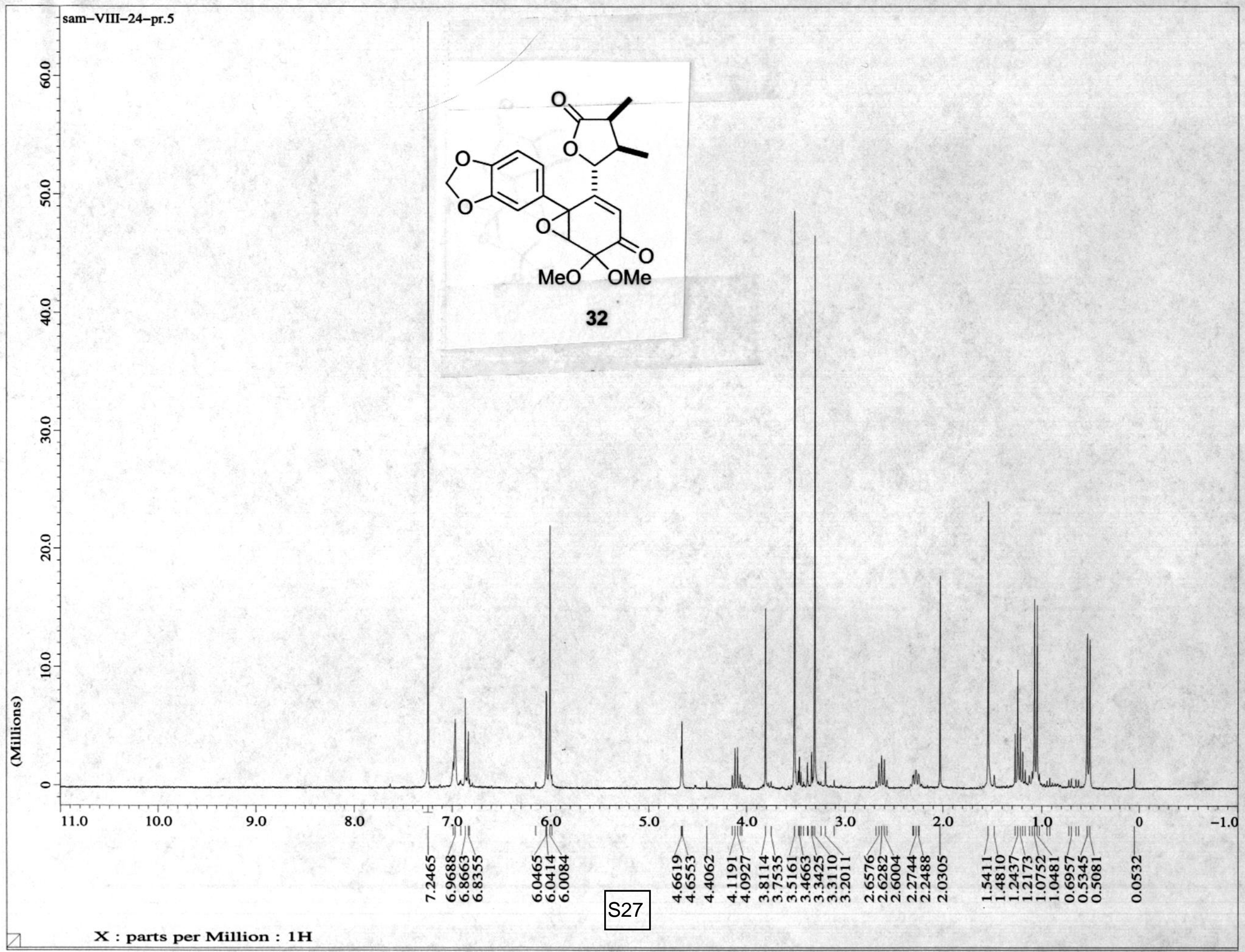




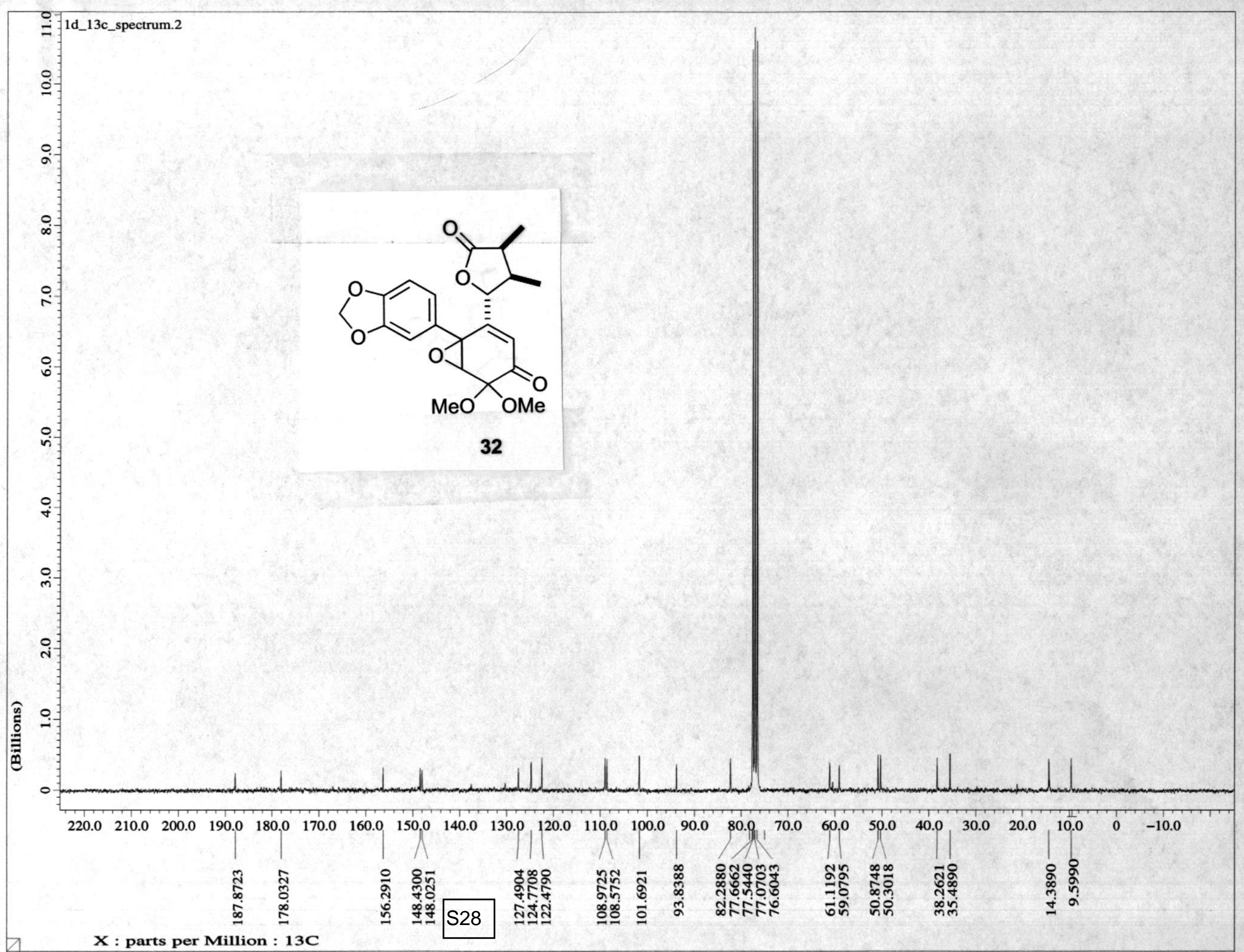




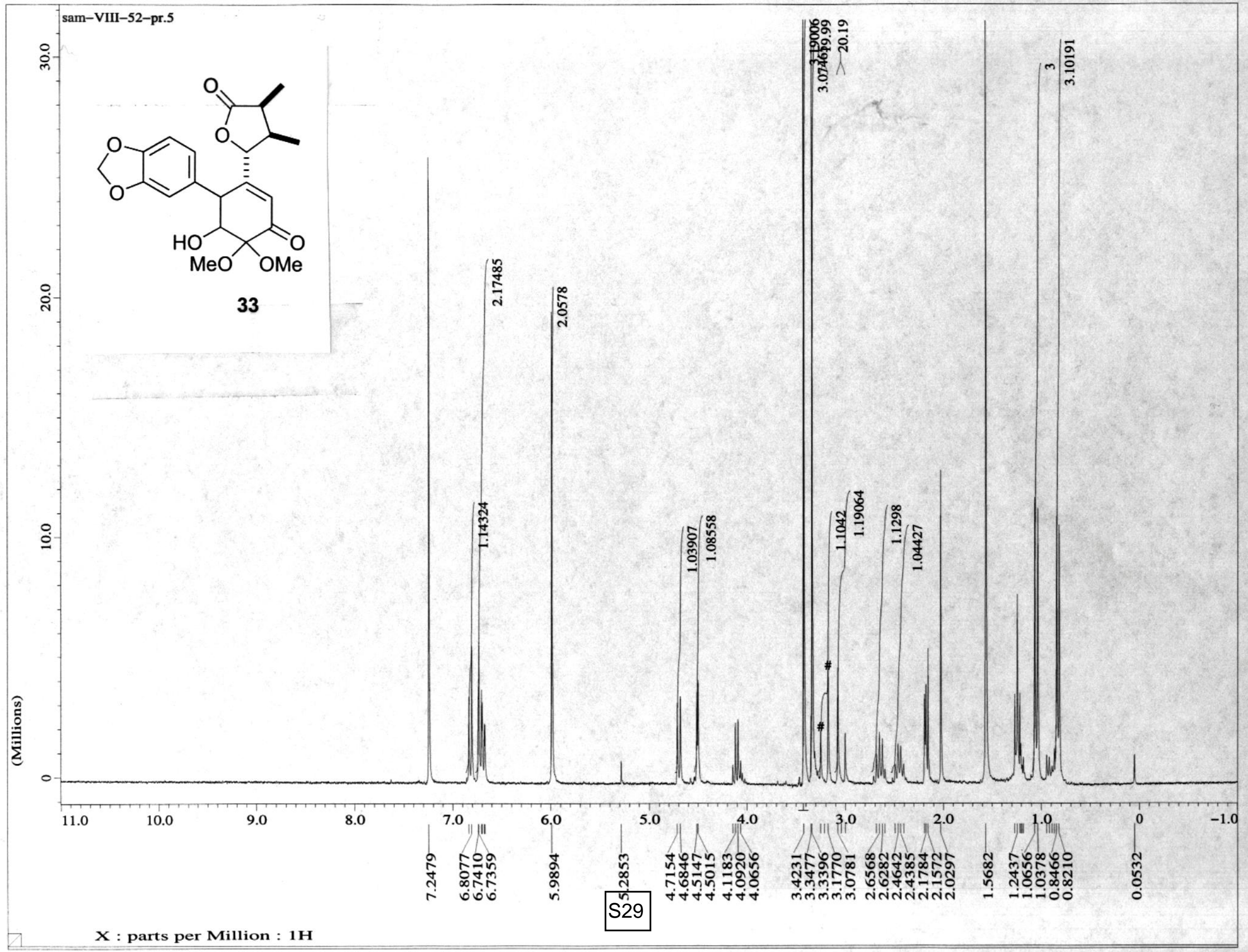




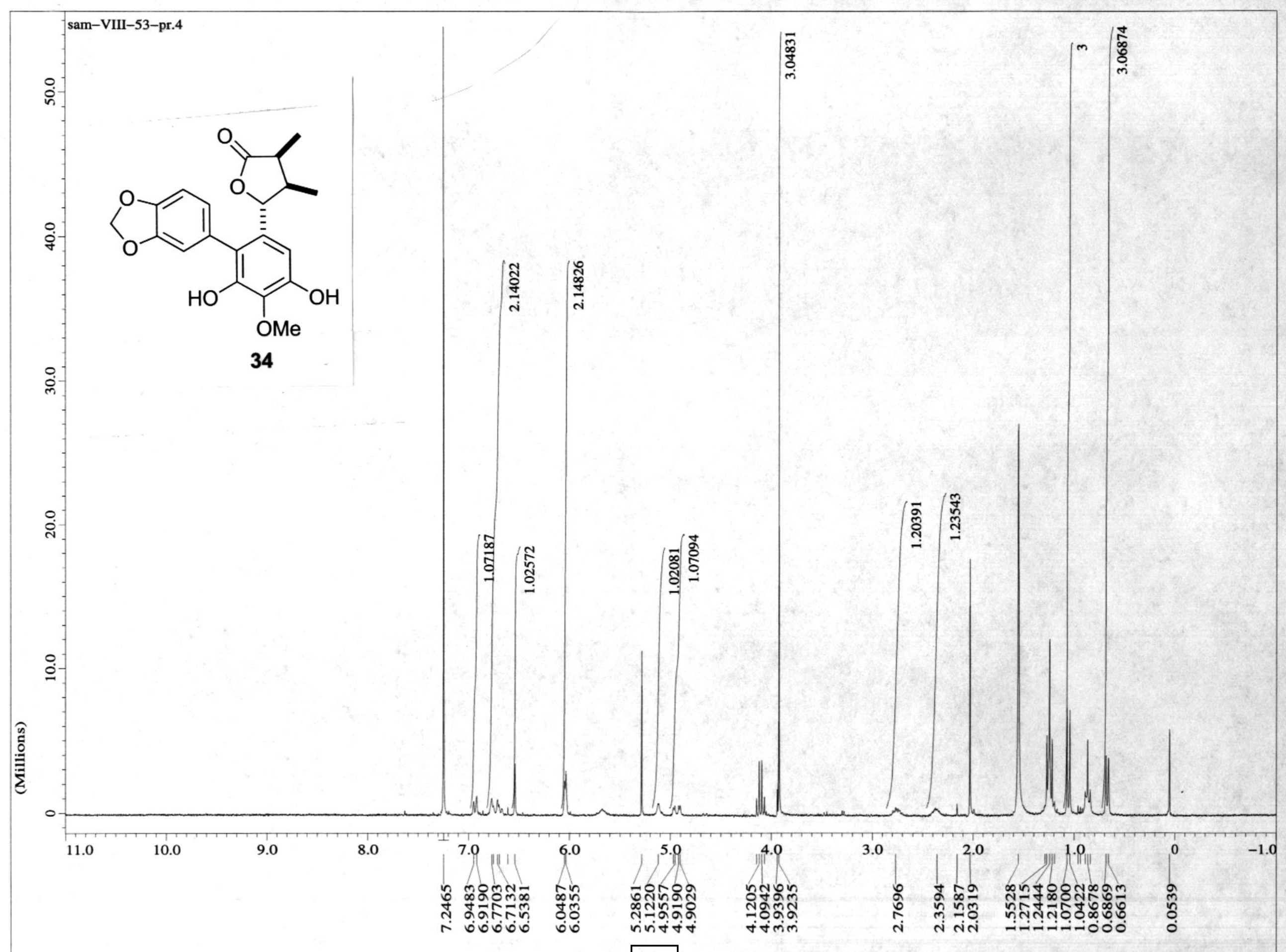

S30 


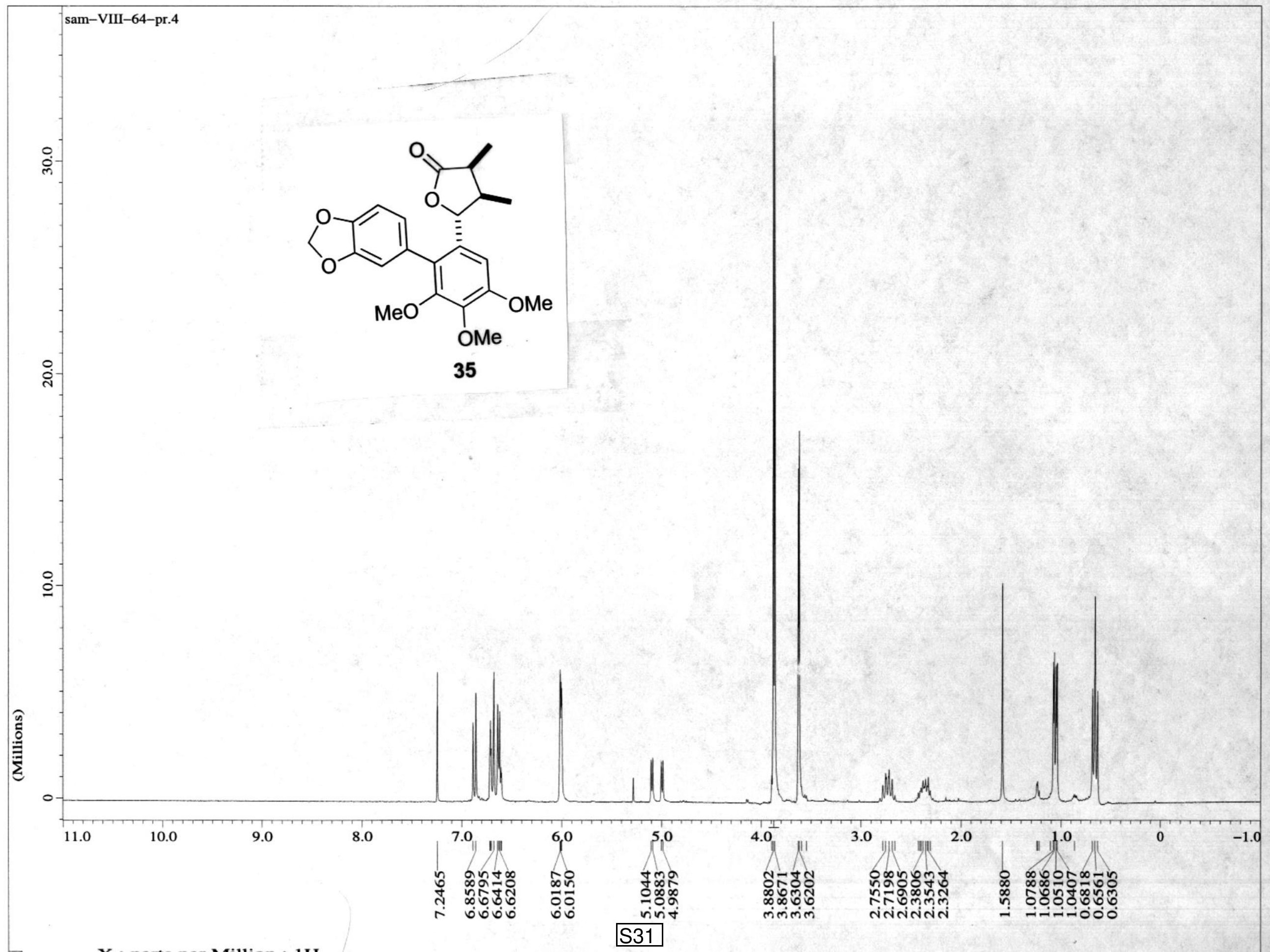




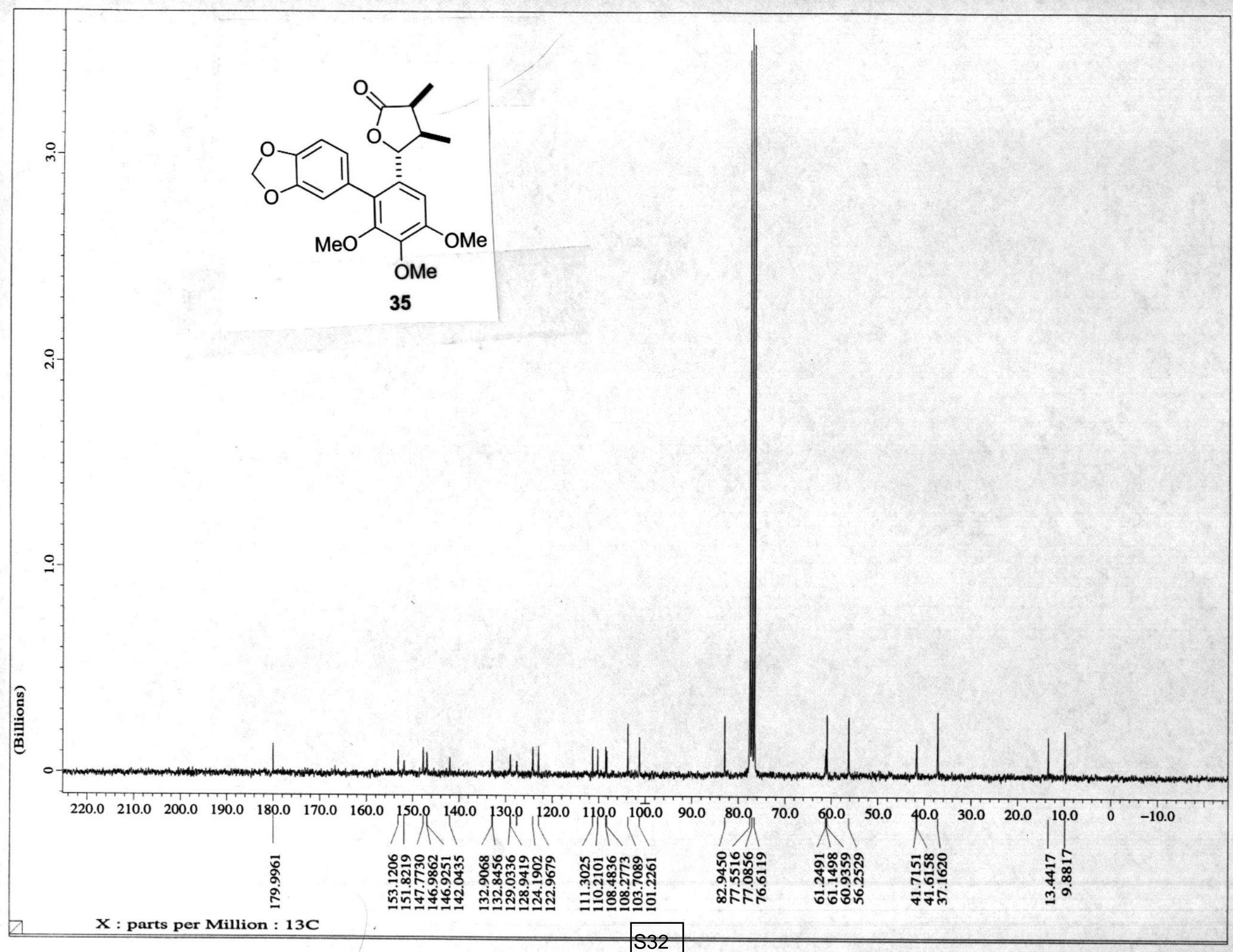




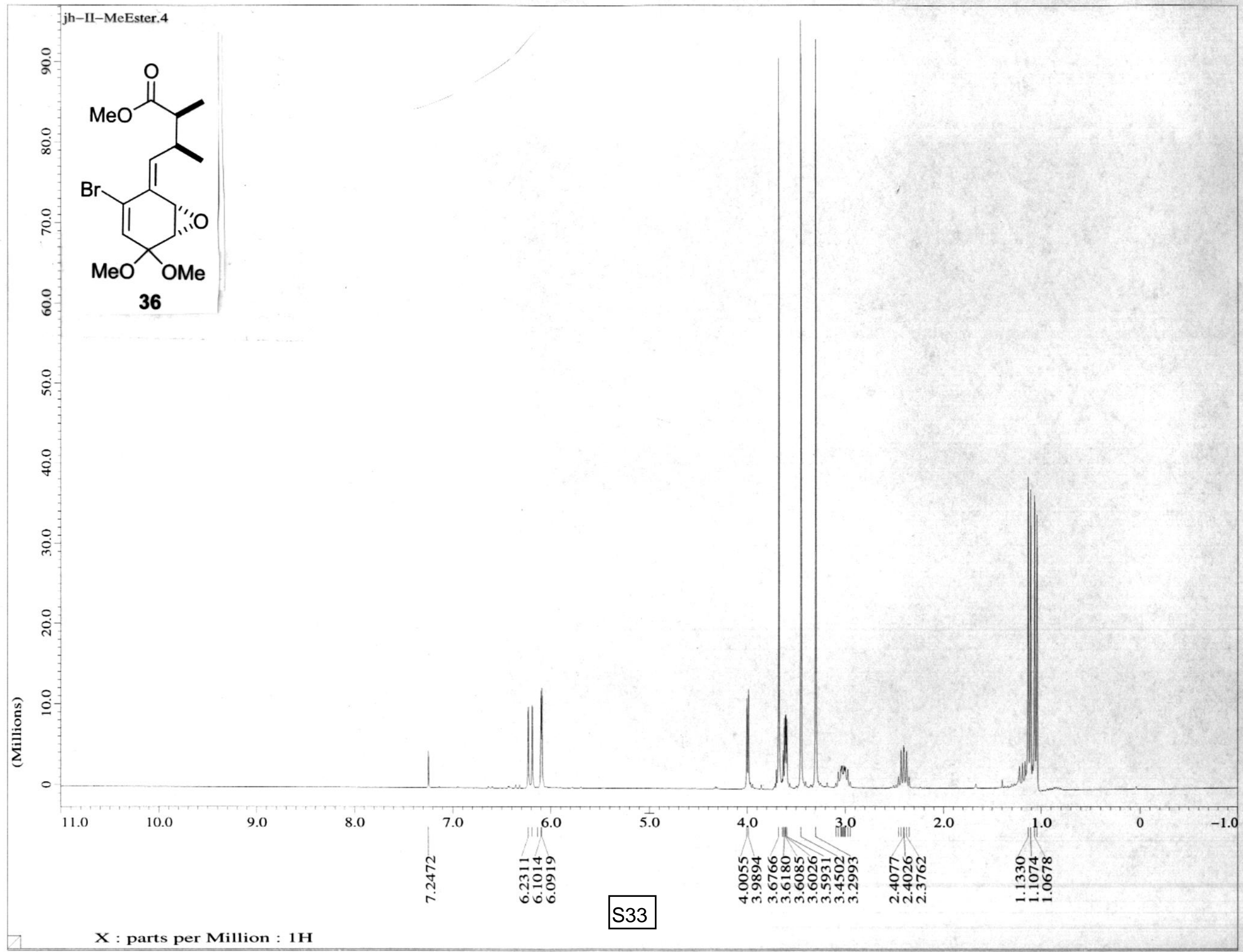



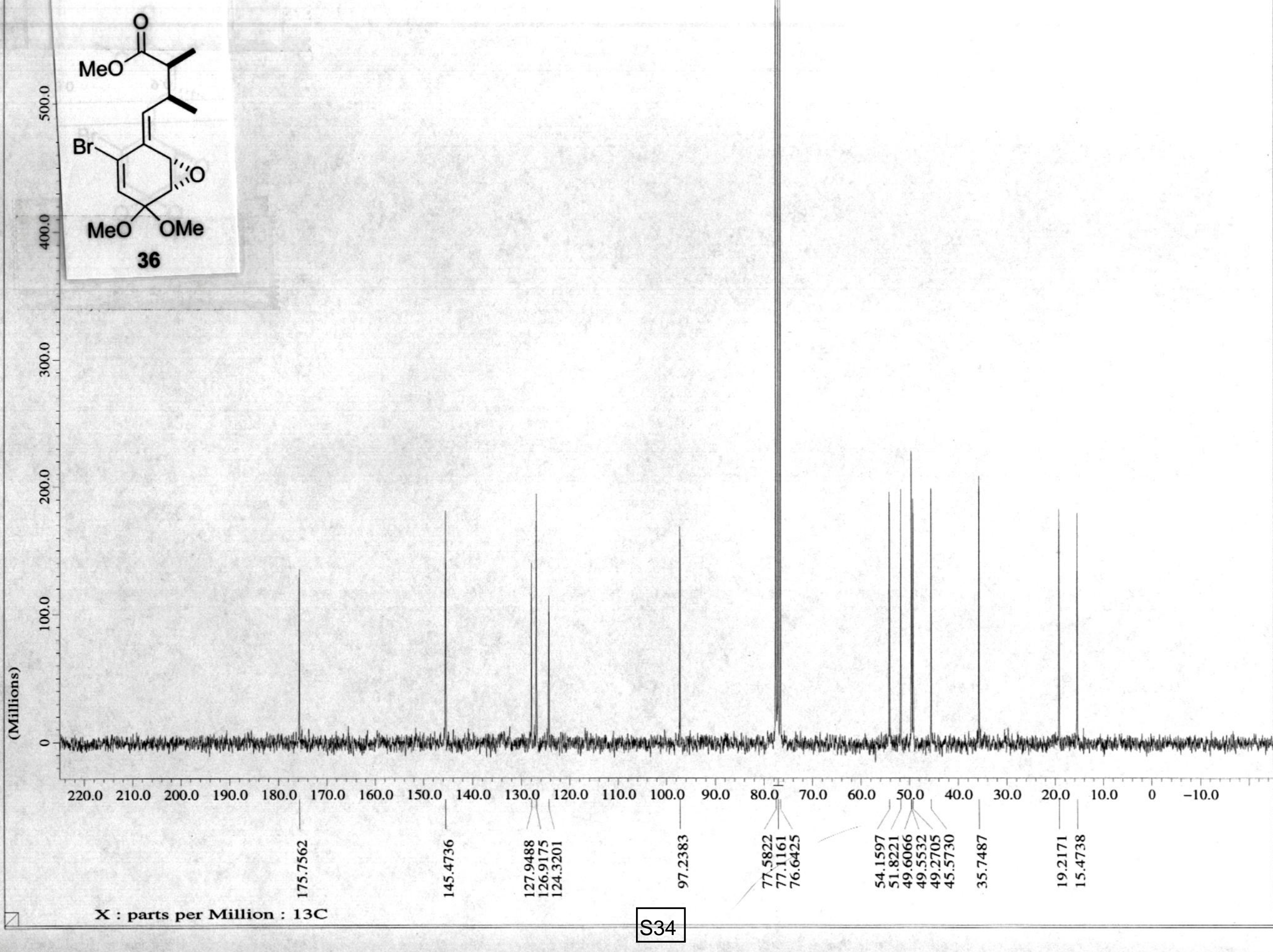


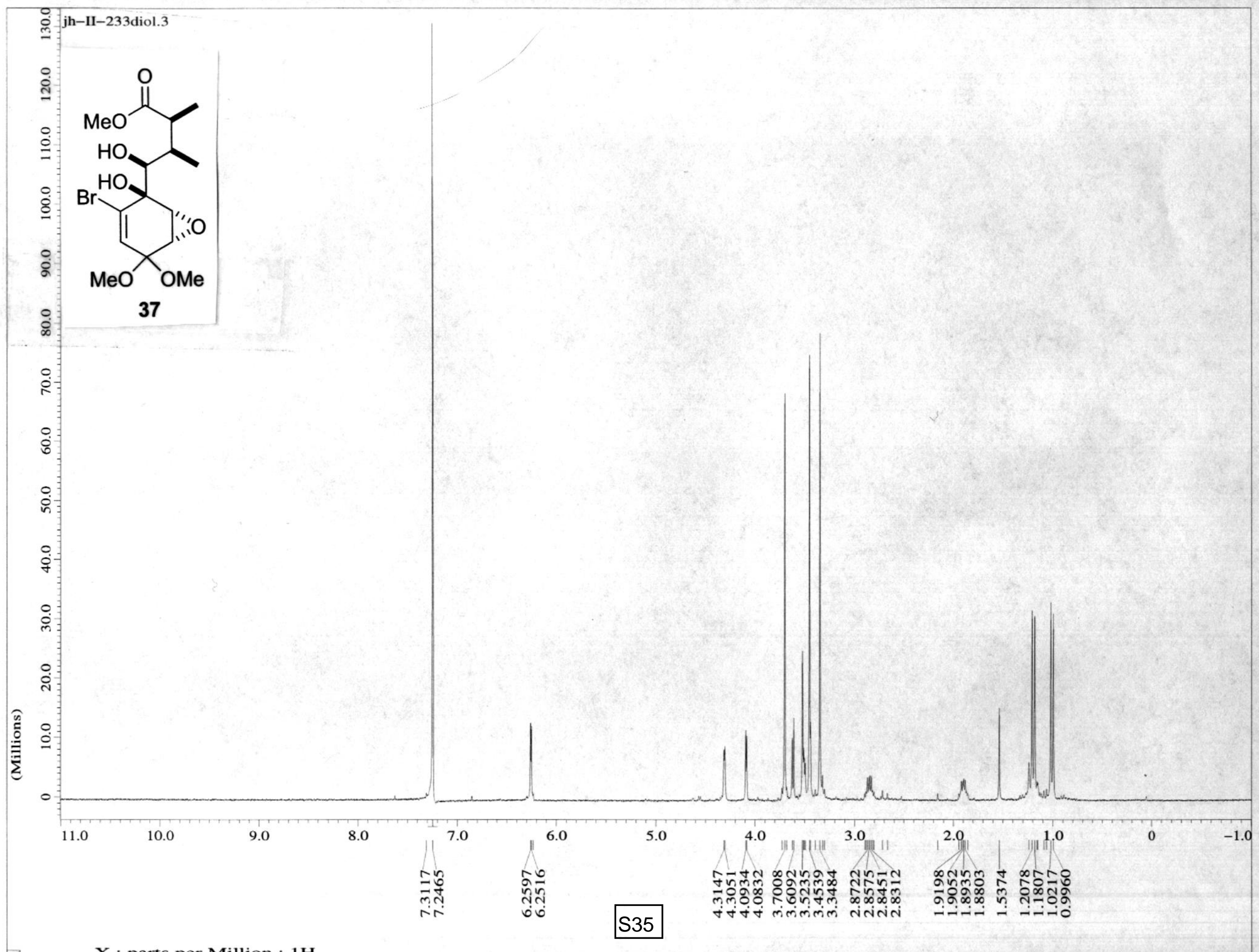

$X$ : parts per Million : $1 \mathbf{H}$ 


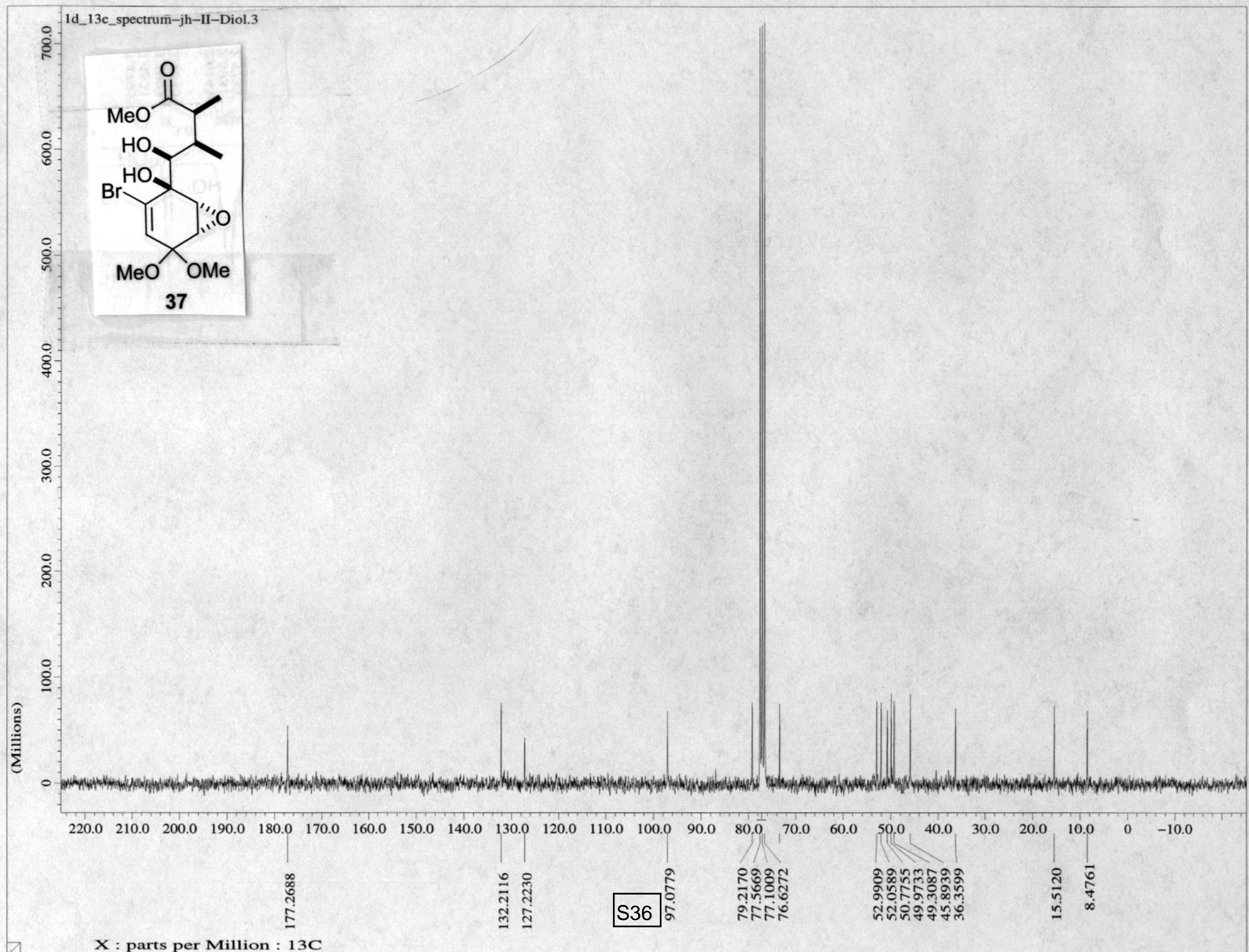




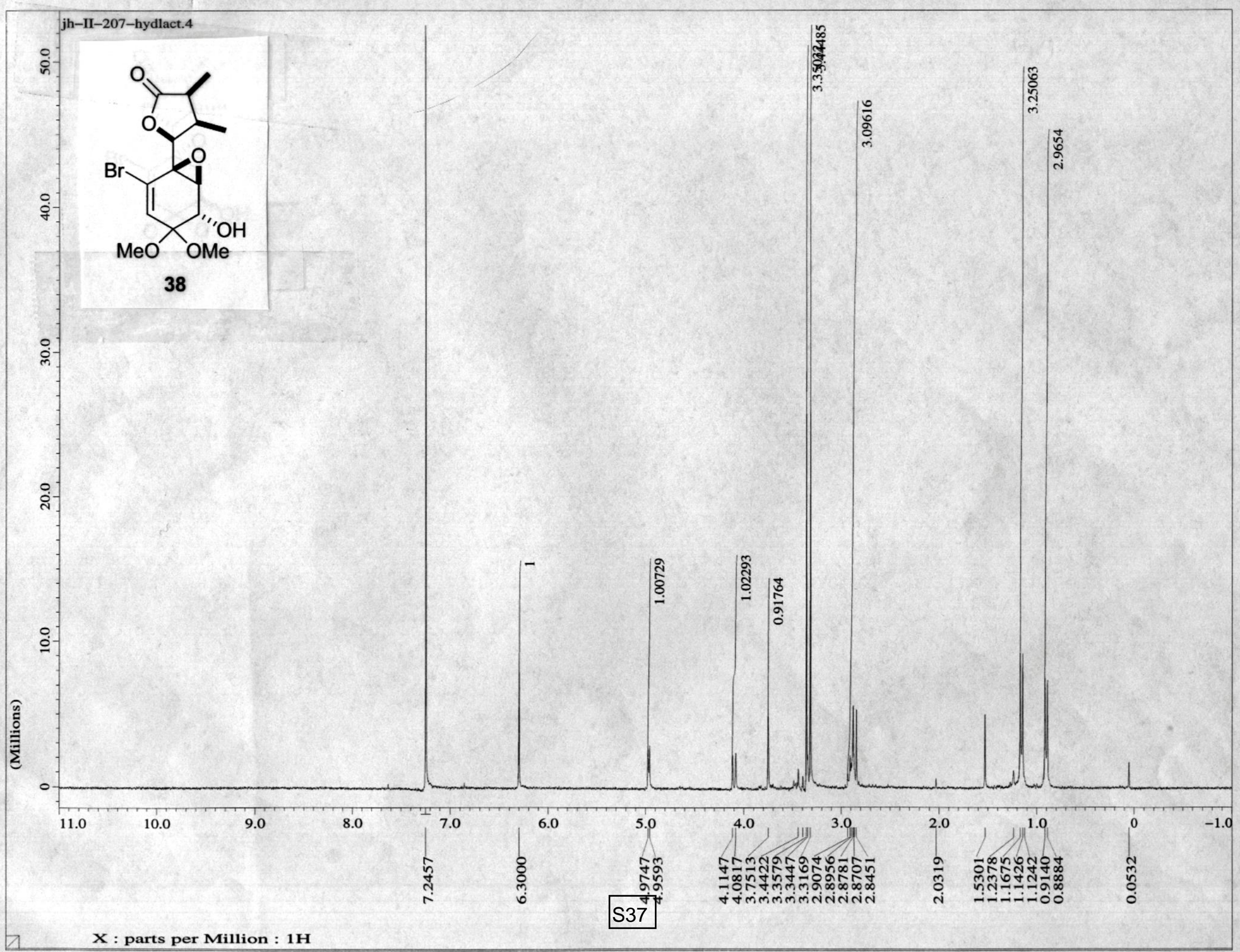




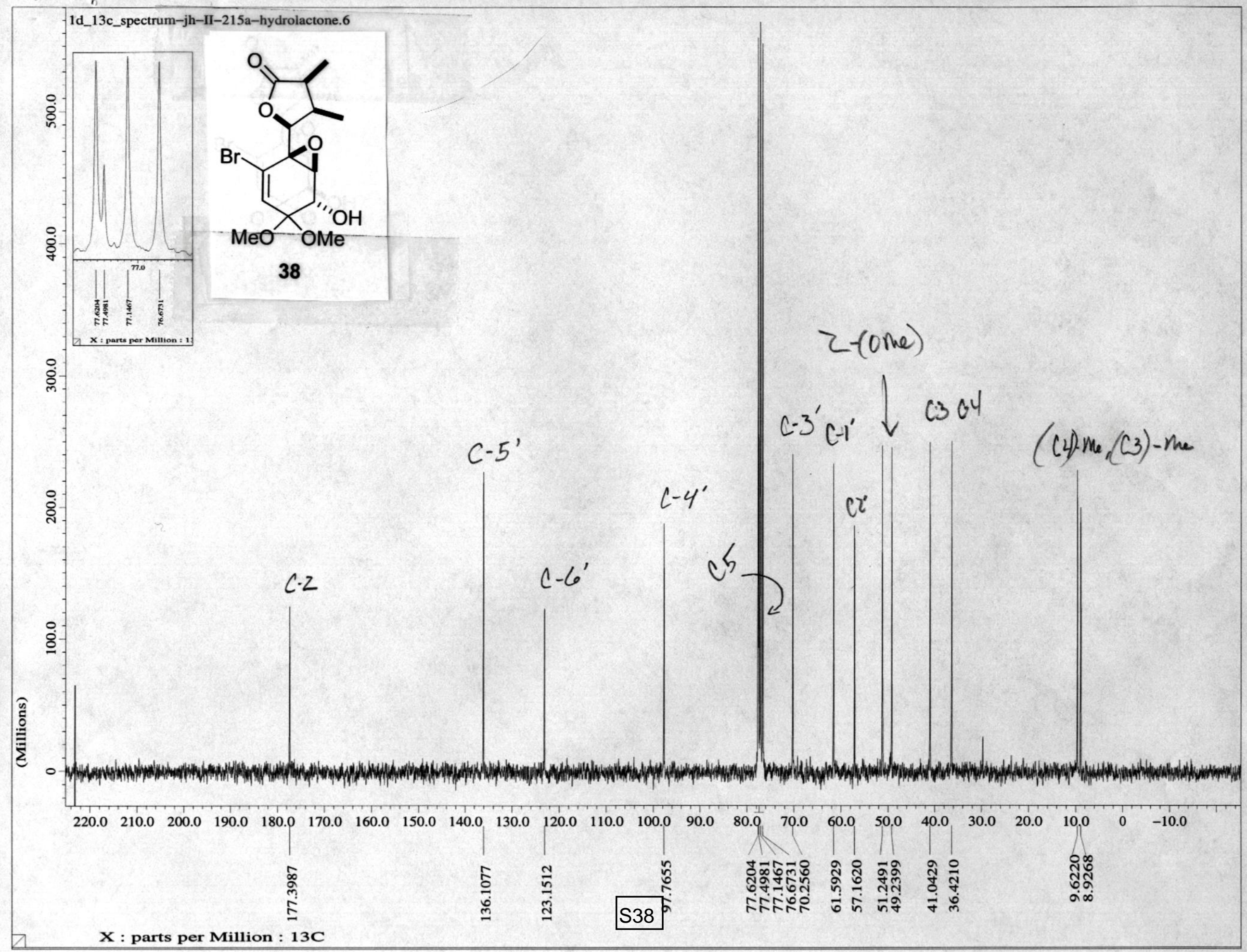




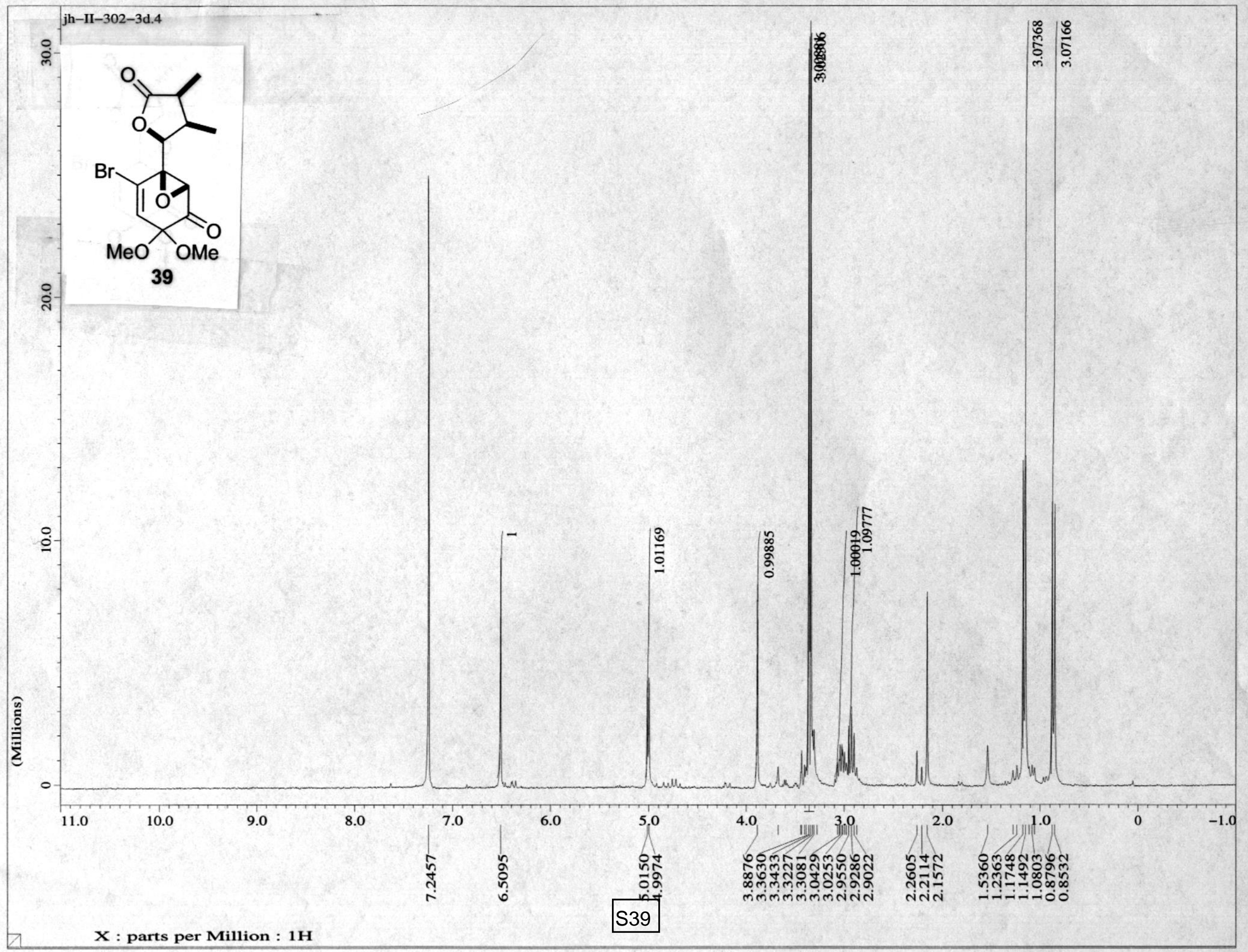




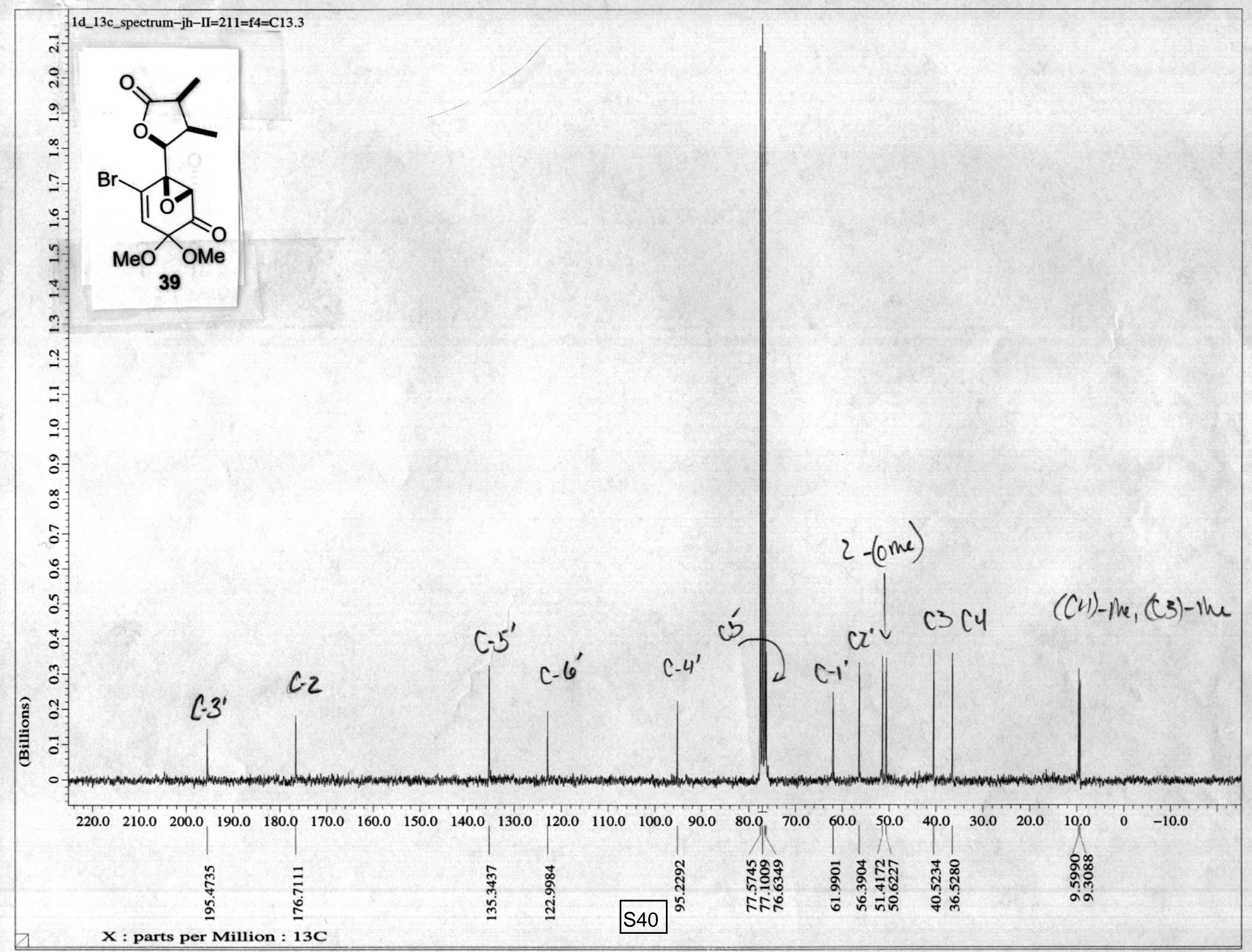




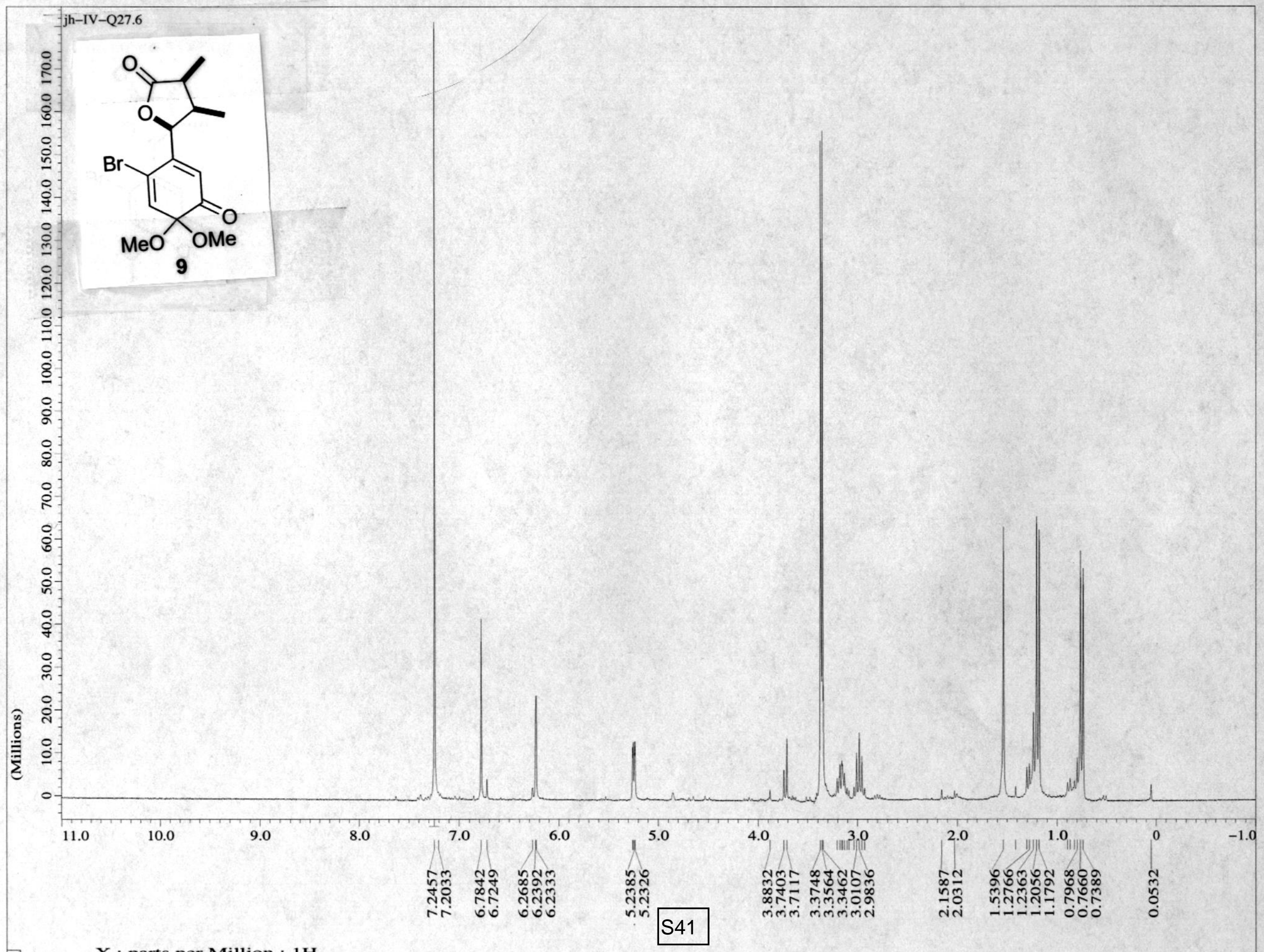




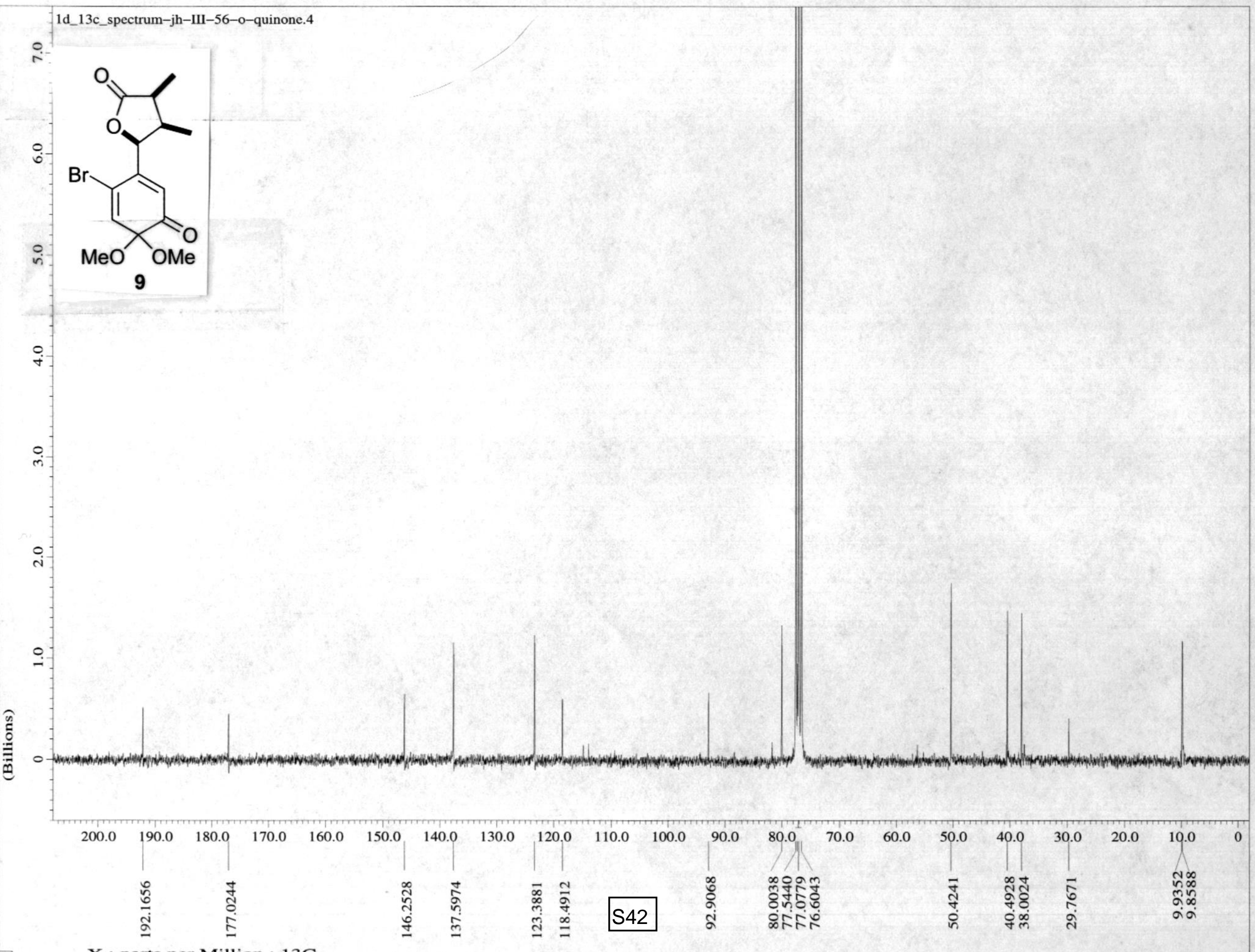

